\title{
Indole-Based Small Molecules as Potential Therapeutic Agents for the Treatment of Fibrosis
}

\author{
Rui Qin ${ }^{1 \dagger}$, Qian Zhao ${ }^{1 \dagger}$, Bo Han ${ }^{1}$, Hong-Ping Zhu ${ }^{1,2}$, Cheng Peng ${ }^{1}$, Gu Zhan ${ }^{1 *}$ and \\ Wei Huang ${ }^{1 *}$
}

${ }^{1}$ State Key Laboratory of Southwestern Chinese Medicine Resources, Hospital of Chengdu University of Traditional Chinese Medicine, School of Pharmacy, Chengdu University of Traditional Chinese Medicine, Chengdu, China, ${ }^{2}$ Antibiotics Research and Re-Evaluation Key Laboratory of Sichuan Province, Sichuan Industrial Institute of Antibiotics, Chengdu University, Chengdu, China

Indole alkaloids are widely distributed in nature and have been particularly studied because of their diverse biological activities, such as anti-inflammatory, anti-tumor, anti-bacterial,

OPEN ACCESS

Edited by:

Syed Nasir Abbas bukhari, Al Jouf University, Saudi Arabia

Reviewed by: Milad Ashrafizadeh, Sabancı University, Turkey Ngoc-Van Nguyen, Can Tho University of Medicine and Pharmacy, Vietnam

*Correspondence:

Wei Huang huangwei@cdutcm.edu.cn

Gu Zhan

zhangu@cdutcm.edu.cn

${ }^{\dagger}$ These authors have contributed equally to this work and share first authorship

Specialty section:

This article was submitted to Experimental Pharmacology and Drug

Discovery,

a section of the journal

Frontiers in Pharmacology

Received: 30 December 2021

Accepted: 19 January 2022

Published: 16 February 2022

Citation:

Qin R, Zhao Q, Han B, Zhu H-P,

Peng C, Zhan G and Huang W (2022)

Indole-Based Small Molecules as

Potential Therapeutic Agents for the

Treatment of Fibrosis.

Front. Pharmacol. 13:845892.

doi: 10.3389/fphar.2022.845892 and anti-oxidant activities. Many kinds of indole alkaloids have been applied to clinical practice, proving that indole alkaloids are beneficial scaffolds and occupy a crucial position in the development of novel agents. Fibrosis is an end-stage pathological condition of most chronic inflammatory diseases and is characterized by excessive deposition of fibrous connective tissue components, ultimately resulting in organ dysfunction and even failure with significant morbidity and mortality. Indole alkaloids and indole derivatives can alleviate pulmonary, myocardial, renal, liver, and islet fibrosis through the suppression of inflammatory response, oxidative stress, TGF- $\beta / S m a d$ pathway, and other signaling pathways. Natural indole alkaloids, such as isorhynchophylline, evodiamine, conophylline, indirubin, rutaecarpine, yohimbine, and vincristine, are reportedly effective in organ fibrosis treatment. In brief, indole alkaloids with a wide range of pharmacological bioactivities are important candidate drugs for organ fibrosis treatment. The present review discusses the potential of natural indole alkaloids, semi-synthetic indole alkaloids, synthetic indole derivatives, and indole-contained metabolites in organ fibrosis treatment.

Keywords: indole alkaloids, organ fibrosis, mechanisms, TGF- $\beta /$ Smad pathway, extracellular matrix

\section{INTRODUCTION}

Indole alkaloids are bicyclic nitrogenous compounds formed by the combination of six-membered benzene and five-membered pyrrole. Such alkaloids are widely distributed in the plant families. They mainly exist in Leguminosae, Loganiaceae, Apocynaceae, Clavicipitaceae, and Rubiaceae (Martins and Nunez, 2015; Zhu et al., 2015). They are the active ingredients of many medicinal plants and have diverse biological activities, such as anti-tumor, anti-inflammatory, and antibacterial, which play important roles in our lives (Ishikawa et al., 2009; Keglevich et al., 2012; Sravanthi and Manju, 2016; Chadha and Silakari, 2017; Singh and Singh, 2018). Well-known natural and synthetic drugs characterized by indole frameworks are very common (Figure 1; Mori et al., 2003; Sasaki et al., 2010; Henriksbo et al., 2014; Abdelfatah and Efferth, 2015; Beuselinck et al., 2015; Soria et al., 2018). The structural modification of these active molecules to screen compounds with novel structure and higher activity is among the hot spots in pharmaceutical chemistry research. Recently, a series of natural, semi-synthetic, synthetic compounds and metabolites that characterize the indole portion of 


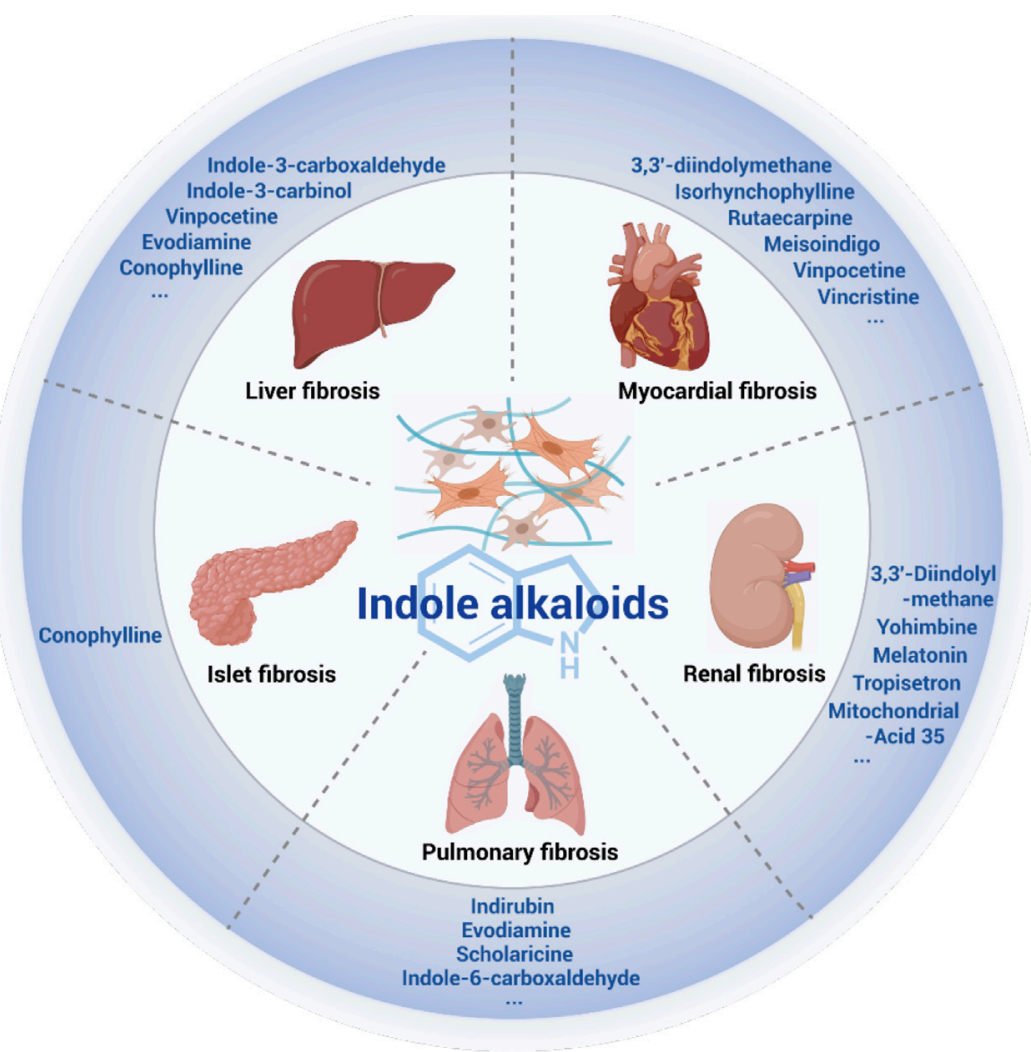

GRAPHICAL ABSTRACT |

their structures have been used to explore the functional role of indole alkaloids against organ fibrosis, many of which have shown promising outcomes. Indole alkaloids have powerful therapeutic effects on various fibrotic diseases. However, their mechanisms of action are complex and may be related to many signaling pathways in the fibrosis process.

Fibrosis is a highly dynamic process and results from the abnormal regulation of the tissue repair response following multiple types of tissue injury, especially during chronic inflammatory diseases (Wynn and Ramalingam, 2012; Henderson et al., 2020). Fibrosis formation is defined as excessive deposition of extracellular matrix (ECM) components such as collagen and fibronectin, which can affect nearly every organ system, including skin, heart, liver, lung, kidney, and pancreas. In case of sustained or severe injury, ECM components continue to accumulate, leading to structural destruction and dysfunction of organs, and even failure (Kendall and Feghali-Bostwick, 2014; Weiskirchen et al., 2019). In the COVID-19 outbreak at the end of 2019, most of the infected patients developed the sequelae of pulmonary fibrosis, which is one of the health issues we are most concerned about (George et al., 2020). In a study on the inhibition of fibrosis, it was found that although the pathogenesis of fibrosis in different organs is complex and different, the basic common process involves inflammatory stimulation and organ parenchymal cell necrosis, which stimulate the activation of macrophages and monocytes, as well as excessive production of cytokines and chemokines (including TGF- $\beta$, CYGF, PDGF, IL-1 $\beta$, IL-6, IL-11, and TNF- $\alpha$ ), ultimately leading to fibrosis (Rockey et al., 2015; Horowitz and Thannickal, 2019). In addition, some non-peptide mediators, such as reactive oxygen species (ROS) and lipid mediators, can also induce fibrogenesis (Sanchez-Valle et al., 2012).

Excessive studies had shown that TGF- $\beta 1 /$ Smad pathway was an important pathogenic mechanism in organ fibrosis. Smads proteins are downstream signal transducers of TGF- $\beta$. TGF- $\beta$ activates Smad 2 and Smad 3 after binding to its receptor and then forms a complex with Smad4 to enter the nucleus and bind to target genes to cause ECM production (Chen et al., 2018; Zhang et al., 2020a). Besides, activation of the nuclear factor kappa B (NF- $\kappa \mathrm{B})$ pathway can increase the expression of various pro-inflammatory factors and thus mediate inflammatory activity. NF- $\mathrm{kB}$ is a heterodimer composed of two subunits $\mathrm{p} 50$ and $\mathrm{p} 65$. When stimulated, I $\mathrm{B}$ is phosphorylated and dissociated from NF- $\kappa \mathrm{B}$ dimer in the presence of protein kinases and phosphatases, revealing nuclear localization signal of p50 protein, thereby activating NF- $\kappa B$. Released NF- $\kappa \mathrm{B}$ translocates to the nucleus, where it binds to specific I $\kappa B$ sequence and triggers the expression of TNF- $\alpha$, IL-6, IL-17, and other pro-inflammatory mediators (Yu et al., 2020; Mirzaei et al., 2021). Sustained activation of Wnt/ $\beta$ catenin pathway is associated with the pathogenesis of fibrotic 

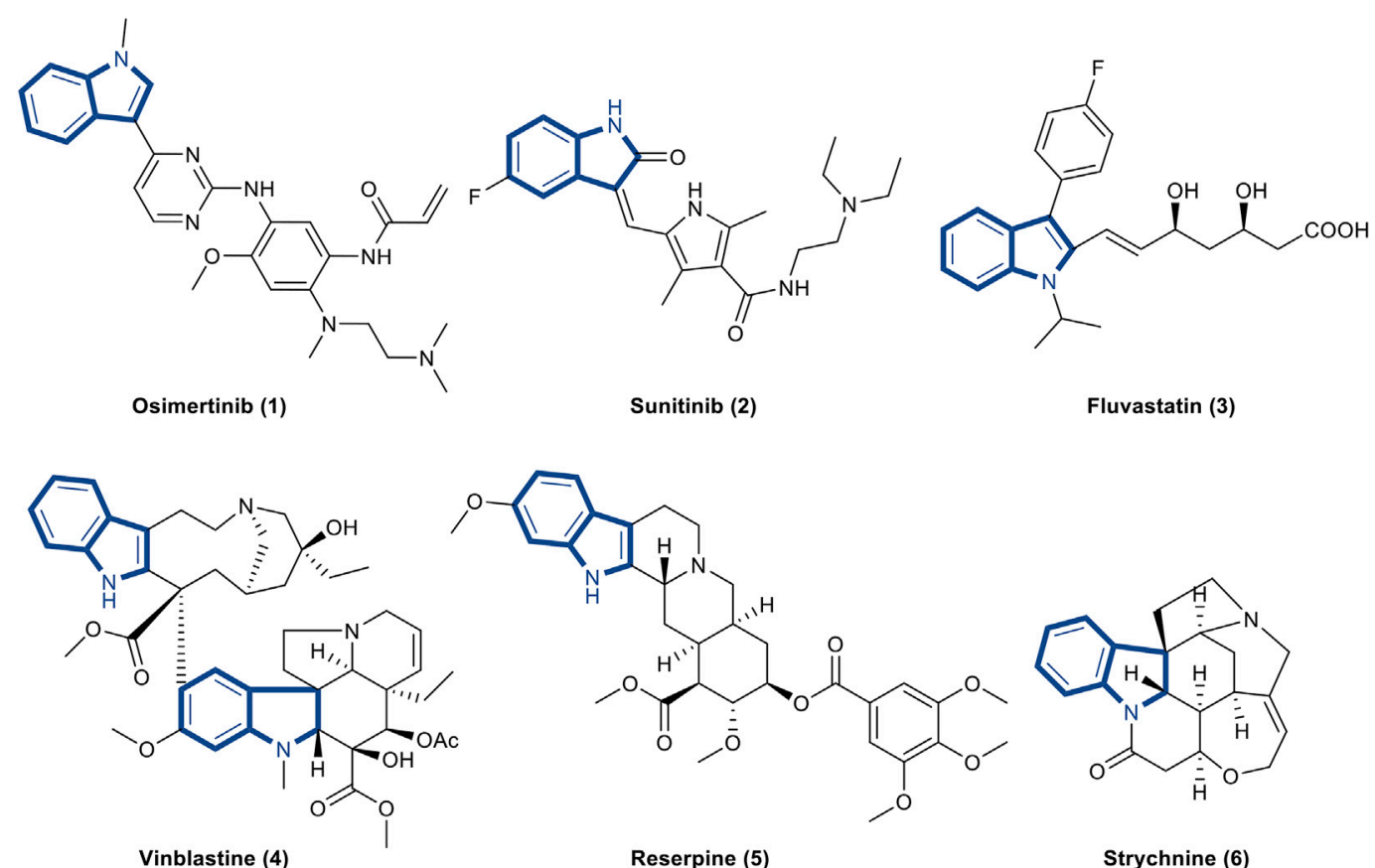

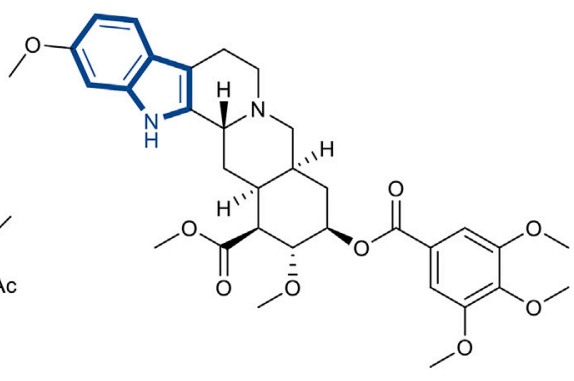

Reserpine (5)

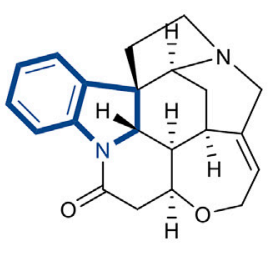

Strychnine (6)

FIGURE 1 | Representative drugs containing indole skeleton. (1). Osimertinib is the first and only NSCLC drug approved for the EGFR T790M mutation (Soria et al., 2018). (2). Sunitinib is a new multi-targeted tyrosine kinase inhibitor for the treatment of tumors (Beuselinck et al., 2015). (3). Fluvastatin is a hydroxymethylglutaryl-CoA (HMG-CoA) reductase inhibitor and a fully synthetic blood lipid lowering drug (Henriksbo et al., 2014). (4). Vinblastine is an antitumor drug extracted from Catharanthus roseus that interferes with protein synthesis (Sasaki et al., 2010). (5). Reserpine is an antihypertensive drug found in the roots of Rauwolfia serpentine (Abdelfatah and Efferth, 2015). (6). Strychnine is an indole alkaloid extracted from Strychnos that excites the spinal cord and enhances skeletal muscle tone Mori et al., 2003.

disorders. When the Wnt pathway is activated, the degradation of free $\beta$-catenin in cytoplasm is inhibited and the content of $\beta$ catenin is increased. $\beta$-catenin enters the nucleus and polymerizes with $\mathrm{T}$ cell factors and lymphocyte enhancer factors (TCF/LEF) to form intranuclear complexes, which regulate transcription of target genes and ultimately accelerate the progression of fibrosis (Wang et al., 2018; Schunk et al., 2021). In addition to the above-mentioned pathways, Raf/MEK/ERK, JAK/STAT and other pathways are also involved in the regulation of fibrosis (Figure 2; Guo et al., 2018; Foglia et al., 2019; Liu et al., 2019a; Pompili et al., 2019; Montero et al., 2021). Fibrous diseases reportedly cause up to $45 \%$ of deaths in the western developed country (Oruqaj et al., 2015). To date, few drugs have been approved to treat fibrosis, and these drugs can only provide relief and cannot fundamentally reverse or cure the disease. Therefore, it is necessary to further study the pathogenesis of fibrosis and find ideal pharmaceuticals to control it.

Indole is a privileged scaffold in anti-fibrotic drug discovery. Recently, due to the high biological activity of indole alkaloids, many studies have been conducted to explore its anti-fibrosis effect. Indole-based small molecules can improve pulmonary, liver, myocardial, renal, and islet fibrosis by regulating the NF- $\kappa \mathrm{B}$ pathway, Wnt/ $\beta$-catenin cascade, TGF- $\beta /$ Smad pathway, Nrf- $2 /$ HO- 1 cascade, PTEN/AKT signaling pathway, and so on. The use of indole alkaloids for the treatment of organ fibrosis is promising. The aim of this review is to verify the therapeutic effects and activities of indole alkaloids and its derivatives on organ fibrosis.

\section{Pulmonary Fibrosis}

Pulmonary fibrosis is a chronic interstitial lung disease caused by a variety of internal and external pathogenic factors. It is characterized by the infiltration of inflammatory cells, the proliferation of fibroblasts, and the deposition of fibrous connective tissue in the lung interstitium (Todd et al., 2012; Rajasekaran et al., 2015; Chanda et al., 2019). The COVID-19 outbreak at the end of 2019 is one of the most devastating events in recent years. To date, more than 200 million people worldwide have recovered from COVID-19. However, although the virus has been eradicated, the infected patients still have varying degrees of pulmonary fibrosis complications, which can cause respiratory failure. In severe cases, these complications can lead to death (George et al., 2020). Several clinical, radiological, and histopathological research data indicated that secondary pulmonary fibrosis will threaten survival and cause exacerbation of functional lung damage in patients with severe COVID-19 (Lin et al., 2020; Schwensen et al., 2020; Tian et al., 2020; Zhou et al., 2020). At present, pirfenidone and nintedanib are the most promising drugs for the treatment of COVID-19induced pulmonary fibrosis. Nintedanib is a transforming growth factor- $\beta$ (TGF- $\beta$ ) inhibitors, as well as a chemosynthetic indole alkaloid. This is a breakthrough drug in the treatment of pulmonary fibrosis. Oral nintedanib can slow the progression 


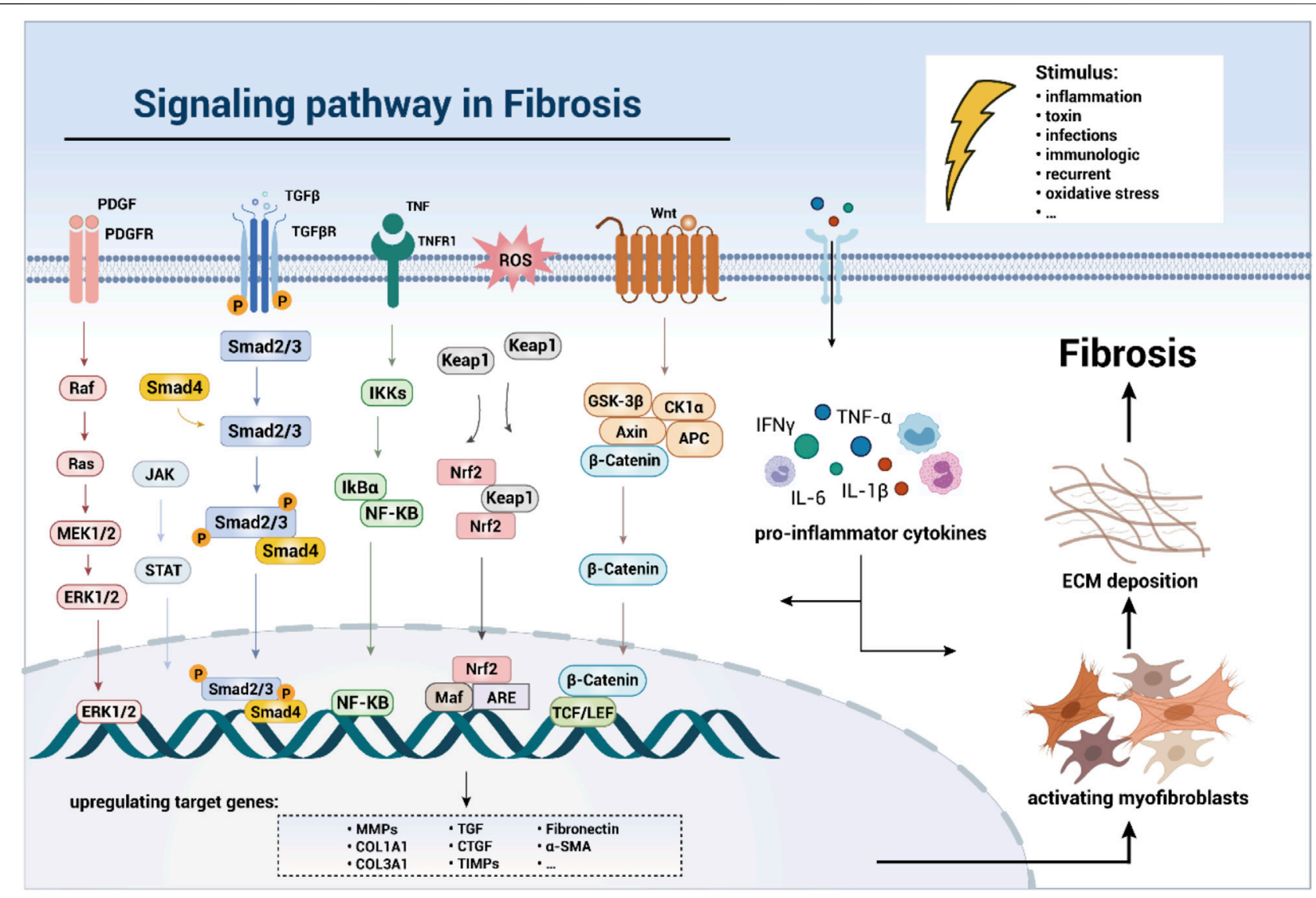

FIGURE 2 | Schematic diagram of the interaction of profibrotic signaling pathway.

of fibrosis and prolong life, but it has side effects, such as diarrhea and vomiting (Karimi-Shah and Chowdhury, 2015; Kim and Keating, 2015; Wollin et al., 2015). Therefore, with the further study of pulmonary fibrosis, it is extremely urgent to find more efficient and safer drugs to treat pulmonary fibrosis. The occurrence of pulmonary fibrosis is related to the overexpression of IL- $1 \beta$, TNF- $\alpha$, IL- 6 , IL-11, and other profibrotic cytokines and the activation of some inflammatory signaling pathways (Karampitsakos et al., 2017; Li and Kan, 2017). Inhibiting the production of these mediators can significantly alleviate pulmonary fibrosis. At present, pulmonary fibrosis is treated using anti-inflammatory drugs, anti-fibrosis enzyme inhibitors, antioxidant mesenchymal stem cell therapy, and others.

Indirubin, a bis-indole alkaloid, is extracted from indigo plants or mollusks of the Muricidae family. It has anti-tumor and antiinflammatory effects (Gaboriaud-Kolar et al., 2015). Wang et al. investigated the effect of indirubin on bleomycin-induced pulmonary fibrosis in mice. Notably, Indirubin shows protective effect on lungs and can suppress the differentiation of fibroblasts to myofibroblasts in a dose-dependent manner, ultimately reducing BLM-induced lung damage and fibrosis. Indirubin significantly attenuated the expression of fibronectin, collagen I and $\alpha$-SMA by inhibiting TGF- $\beta /$ Smads signaling pathway. Results suggests that indirubin could be a good candidate drug for IPF treatment (Figure 3; Wang et al., 2020). Another indole alkaloid with potential is isorhynchophylline (isorhy), which was isolated from the traditional Chinese herb Tripterygium wilfordii. It reportedly shows anti-inflammatory activities in the nervous and cardiovascular systems (Zhou and Zhou, 2012). Qiu et al. found that isorhy could alleviate SD-induced pulmonary inflammation and fibrosis in mice by suppressing the release of IL-1b, TNF- $\alpha$ and IL- 6 fibrogenic factors and notably reducing collagen deposition in lung tissues. Mice treated with isorhy showed alleviation in body weight loss induced by SD. In addition, isorhy treatment substantially assuaged inflammatory cell infiltration and fibroblast excessive proliferation (Qiu et al., 2020).

Zhao et al. conducted a study on the active ingredients in total alkaloids (TA) and their potential mechanism in the treatment of pulmonary fibrosis. The research suggested that through the analysis of cytokine, histopathological examination and gene expression, the treatment with $50 \mathrm{mg} / \mathrm{kg}$ TA could ameliorate pathological changes in the lung tissue, reduce the content of Krebs von den Lungen-6, TGF- $\beta$, collagen I and hydroxyproline in lung tissues, and increase the level of superoxide dismutase in the serum (Zhao et al., 2020). According to the above 


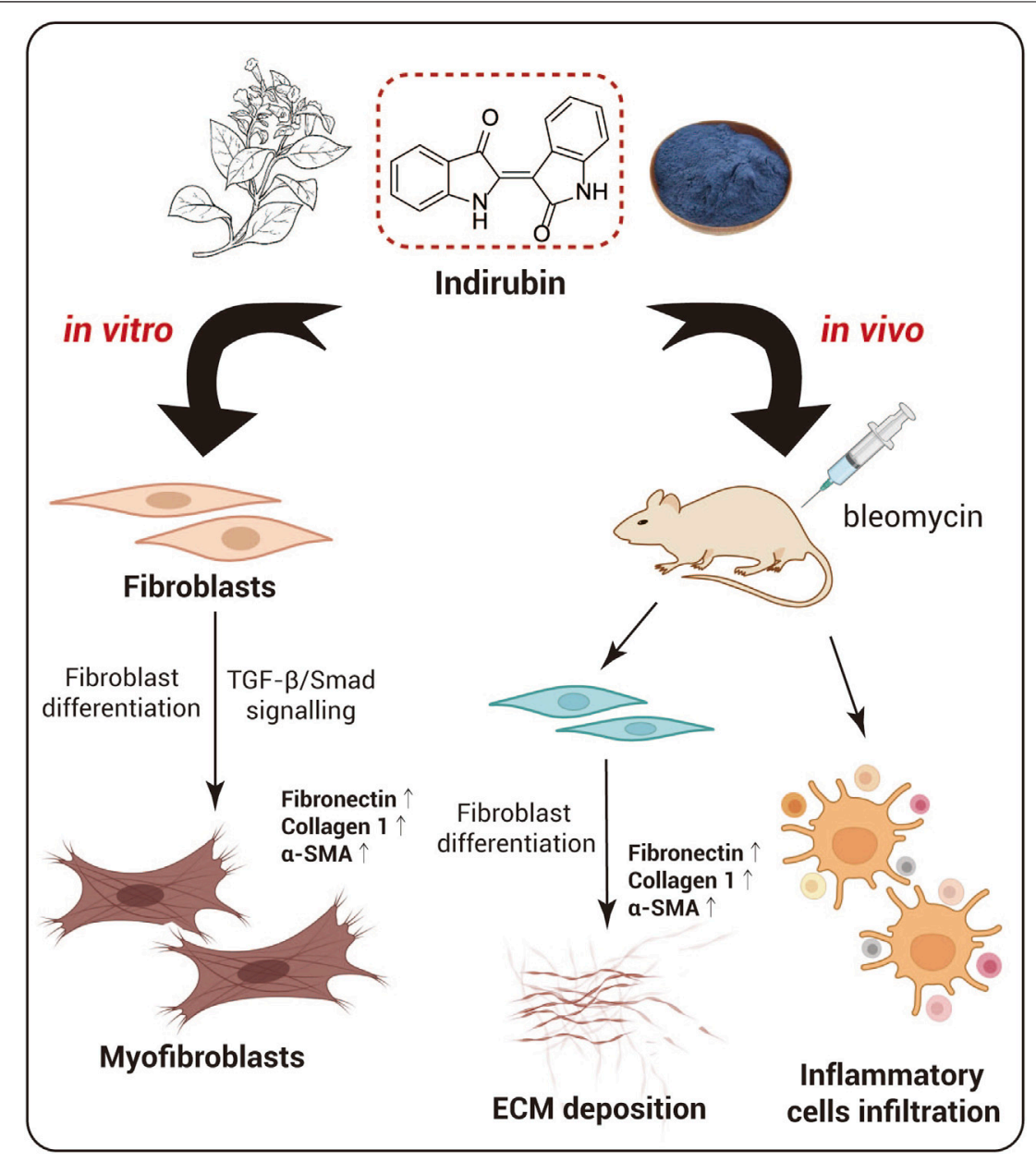

FIGURE 3 | Indirubin ameliorates bleomycin-induced pulmonary fibrosis.

experimental data, the indexes of picrinine and scholaricine are superior to those of other alkaloids; thus, they are the most effective ingredients of TA in the treatment of PF. Overall, TA protected mice against BLM-induced fibrosis by enhancing the expression of TGF- $\beta$ /matrix metalloproteinase-1 (MMP-1) pathway and diminishing the accumulation of collagen (Zhao et al., 2021). Kim et al. reported that I6CA, a natural indole derivative isolated from the marine brown algae Sargassum thunbergia, can protect V79-4 lung fibroblasts from oxidative stress. This group used $\mathrm{H}_{2} \mathrm{O}_{2}$ to induce oxidative damage and found that $\mathrm{H}_{2} \mathrm{O}_{2}$ can stimulate $\mathrm{G} 2 / \mathrm{M}$ cell cycle arrest and DNA damage to reduce V79-4 cell survival. I6CA can reverse the cytotoxicity of $\mathrm{H}_{2} \mathrm{O}_{2}$ by reducing the accumulation of ROS. Additionally, I6CA could significantly promote Nrf2 expression and increase the activity of HO-1. A HO-1 inhibitor, zinc protoporphyrin IX, can suppress HO-1 activity and eradicate the ROS scavenging activity of I6CA, as well as prevent anti-apoptotic effects of I6CA (Kim and Choi, 2020). In an anti-fibrotic screening of indole alkaloid compound library, $\mathrm{Li}$ et al. found that matrine derivative compound $3 \mathrm{f}$ could attenuate idiopathic pulmonary fibrosis via suppression of fibroblast-to- myofibroblast transition and inhibition of the TGF- $\beta / \mathrm{Smad}$ signaling pathway. Furthermore, compound $3 f$ exhibited approximately 266-fold higher anti-fibrotic activity against MRC-5 cell lines than matrine (Li et al., 2019b).

Ye et al. recently discovered evodiamine in Evodia rutaecarpa; this is an indole alkaloid with excellent anti-inflammatory effect. Evodiamine alleviated inflammation and pulmonary fibrosis induced by lipopolysaccharide (LPS) both in vivo and in vitro (Ye et al., 2021). After treating LPS-induced mice with $10 \mathrm{mg} / \mathrm{kg}$ evodiamine, the apelin level increased significantly, thereby suppressing the increase in the concentrations of IL-6 and CCL17. Therefore, the group speculated that apelin could play a crucial role in the molecular mechanism of evodiamine. In addition, evodiamine could inhibit cell apoptosis and stimulate apelin pathway to regulate the release of inflammatory factors and inhibit the development of inflammation, ultimately attenuating lung fibrosis in LPS-induced mice (Ye et al., 2021). Indole-3carbinol (I3C), an indole alkaloidal compound extracted from cruciferous vegetables, reported that by activating the AhRresponsive genes in rat lungs, the pup's lung injury induced by hyperoxia-hypoxia was alleviated, thereby improving 
alveolarization and decreasing fibrosis. In their experiments, $\mathrm{I} 3 \mathrm{C}$ could activate TNF- $\alpha$ and NF- $\kappa$ B pathway to promote the expressions of VEGF, MCP1, MMP-8, and IL-6 and regulate inflammatory processes. According to results of histopathological examination, the degree of alveolitis and pulmonary fibrosis decreased in the I3C-treated group (Guzman-Navarro et al., 2021).

In a clinical study, nintedanib, a tyrosine kinase inhibitor, was used by Umemura et al. in an interventional trial to assess its efficacy and safety in COVID-19 treatment. The experiment was conducted on adult COVID-19 patients requiring mechanical ventilation (Umemura et al., 2021). The research showed that compared with the control group, the $\mathrm{P} / \mathrm{F}$ ratio of nintedanib group was remarkably increased, the mechanical ventilation time was significantly shortened, and the volume of high-attenuation areas on CT images was notably decreased. These results demonstrated that nintedanib can minimize respiratory sequelae of COVID-19 and improve lung injury by regulating pulmonary fibrosis. Nintedanib can be a novel anti-fibrotic agent approved by the FDA to reduce fibrosis symptoms (Umemura et al., 2021). PXS-5120A is an indole-based fluoroallylamine inhibitor, which could inhibit LOXL2/3 (a secreted enzyme that catalyze the formation of cross-links in collagen and elastin) to treat lung and liver fibrosis. LOXL2 is overexpressed in patients with fibrosis; it usually regulates the TGF- $\beta$ /Smad signaling pathway to activate lung fibroblasts. Therefore, LOXL2 could be a promising therapeutic target for lung fibrosis (Findlay et al., 2019).

\section{Myocardial Fibrosis}

Myocardial fibrosis (MF) is a pathological process in which fibroblasts (CFs) in normal myocardial tissue proliferate and transform into myofibroblasts due to various pathological factors, resulting in excessive deposition of extracellular matrix, increased collagen concentration, and disordered collagen ratio (Kong et al., 2014; Rienks et al., 2014; Pinto et al., 2016). Myocardial fibrosis is closely related to many clinical cardiovascular diseases such as ischemic cardiomyopathy, diabetic cardiomyopathy, hypertensive heart disease, chronic heart failure and hypertrophic cardiomyopathy. As a common pathological result of various diseases, myocardial fibrosis eventually lead to increased myocardial stiffness, decreased ventricular diastolic function, severe ventricular arrhythmias, and even sudden death. According to WHO, cardiovascular disease is the leading cause of death in the world (van Nieuwenhoven and Turner, 2013; Leask, 2015; Kurose and Mangmool, 2016). The occurrence of myocardial fibrosis is closely related to regulatory cytokines (such as TGF- $\beta$, CTGF, and MMP), inflammatory factors (such as TNF- $\alpha$, IL-1 $\beta$, IL-6, and IL-10), oxidative stress and other factors that affect the occurrence and development of myocardial fibrosis by regulating the corresponding signaling pathways.

Yao et al. explored the anti-fibrosis effect of 3,3diindolymethane (DIM) against adriamycin-induced cardiac fibrosis. DIM was obtained from a naturally food additive. Adriamycin possesses strong cardiotoxicity and can cause cardiac fibrosis, eventually leading to heart failure. The DIM

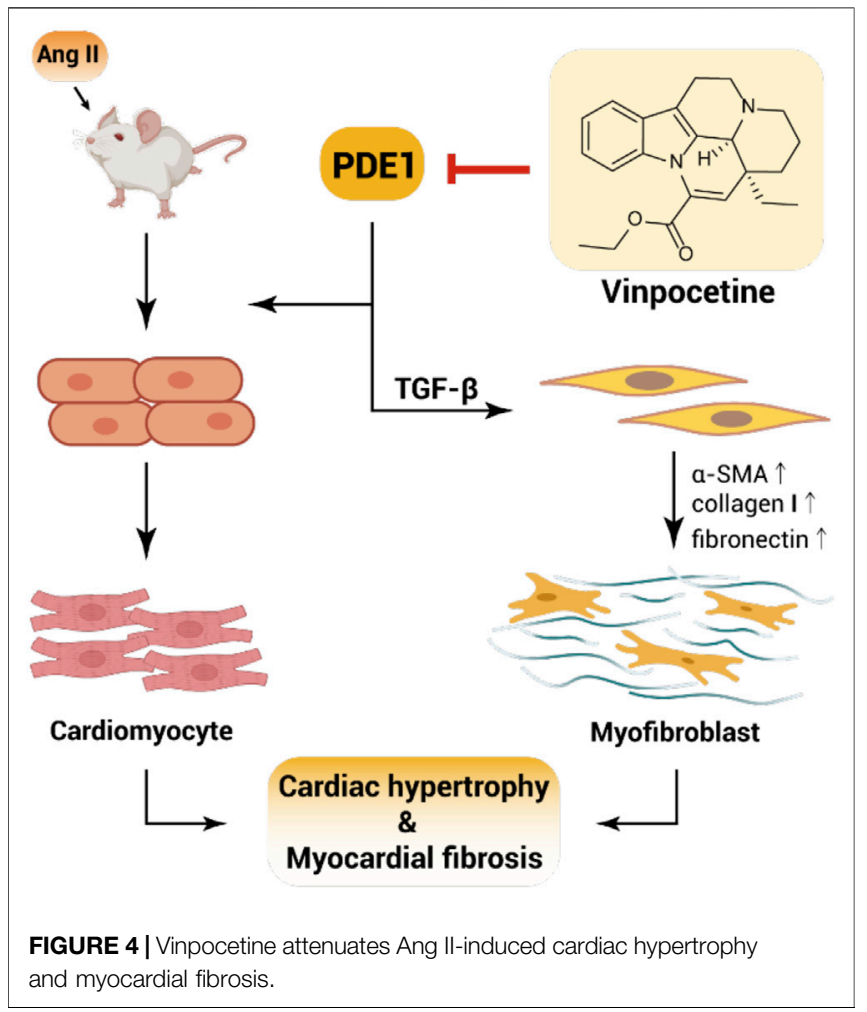

notably showed anti-fibrotic effect on adriamycin-induced cardiac tissue; the effect involved the reduction of collagen I and $a$-SMA, the upregulation of breast cancer type 1 susceptibility protein (BRCA1), and the activation of transcription factor nuclear factor (erythroid-derived 2)-like 2 (Nrf2). Then, DIM alleviated oxidative stress in inflammatory tissues (Yao et al., 2013). Rutaecarpine is an indolopyridoquinazoline alkaloid with powerful cardiovascular effects; it can be isolated from E. rutaecarpa. Li et al. investigated the anti-fibrotic properties of rutaecarpine against right ventricular (RV) remodeling on rats (Li et al., 2016). The experiment data suggested that rutaecarpine could reduce the expression of several related factors (such as $\alpha$-SMA, collagen-I, collagen-III, eIF3a, TGF- $\beta 1$, and others) to attenuate the effect of hypoxic-induced RV remodeling in rats in a dose-dependent manner. Moreover, calcitonin gene-related peptide (CGRP) could attenuate the activity of TGF- $\beta 1$. The results proved that the inhibitory effect of rutaecarpine on hypoxic-induced RV remodeling is stimulated by the release of CGRP, and this effect may also be associated with the eIF3a/p27 pathway (Li et al., 2016).

A study was designed and conducted by $\mathrm{Wu}$ et al. to evaluate the anti-myocardial fibrosis activities of evodiamine, an indole alkaloid isolated from the E. rutaecarpa. Evodiamine could suppress TGF- $\beta 1$-induced activation of adult rat cardiac fibroblasts, which was evidenced by the decrease in the expressions of a-SMA, CTCF, and collagen I/III. In addition, evodiamine could suppress the migration ability of HUVECs (Wu et al., 2017b). Isorhynchophylline (isorhy) is an indole alkaloid obtained from Uncaria rhynchophylla. Zhang et al. 


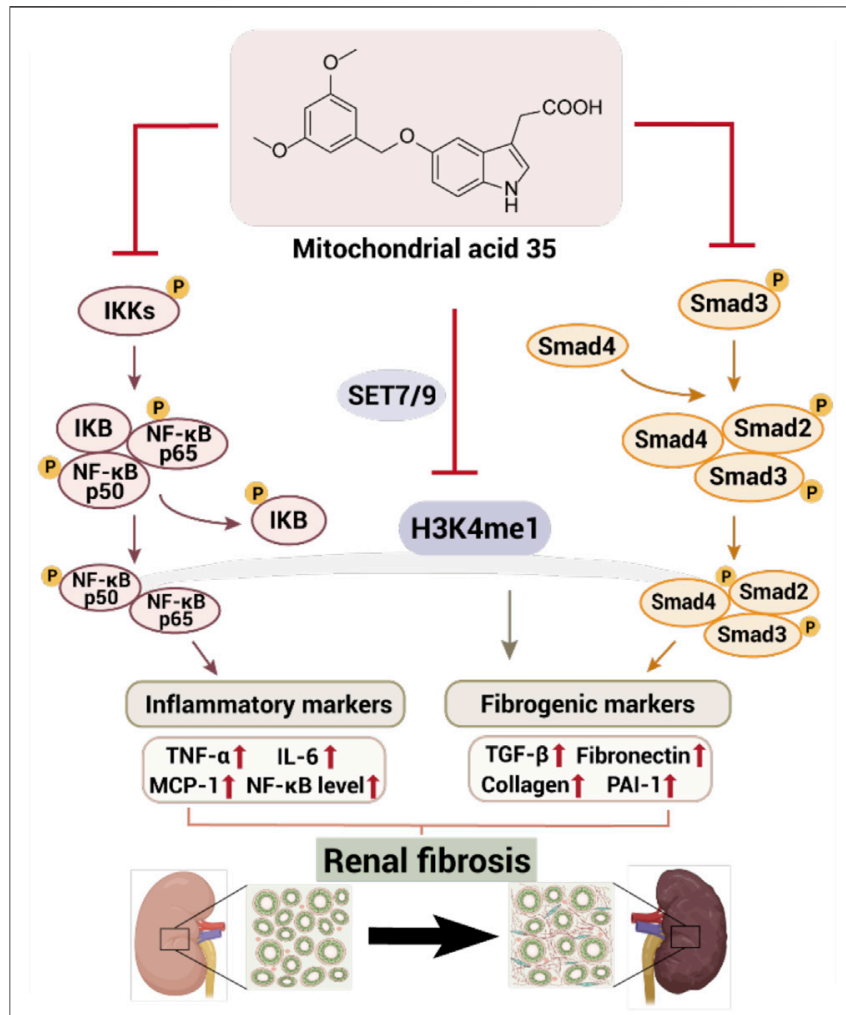

FIGURE 5 | Mitochondrial acid 35 inhibits TNF- $\alpha$ and TNF- $\beta 1$ pathway to ameliorate renal fibrosis.

found that isorhy prevented phenylephrine (PE)-induced myocardial hypertrophy and alleviated myocardial fibrosis in rats by inhibiting the expressions of TGF- $\beta 1$, CTGF, and collagen I/III and other related fibrosis factors. These effects were associated with Nrf2 nuclear translocation and MAPK pathway (Zhang et al., 2020b).

Another study performed by $\mathrm{Wu}$ et al. reported that vinpocetine is a derivative of vincamine alkaloid that is derived from periwinkle plant (Vinca minor). Vinpocetine improved cardiac hypertrophy and fibrosis induced by Ang II infusion (Wu et al., 2017a). The administration of vinpocetine at $5 \mathrm{mg} / \mathrm{kg} /$ day prevented heart enlargement by Ang II. Vinpocetine also inhibited the myocyte hypertrophic growth induced by Ang II in vivo and blocked the activation of fibroblasts and the expression of matrix gene stimulated by TGF- $\beta$ in vitro. PDE1 was a potential molecular target that attenuates cardiac hypertrophy and fibrosis. Vinpocetine possesses critical pharmacological effects and can be used to treat cardiac remodeling and fibrosis (Figure 4, Wu et al., 2017a). Similarly, Liu et al. reported that meisoindigo $(\mathrm{Me})$, a bisindole indirubin derivative, is widely used to treat chronic myelogenous leukemia. A study group assessed whether Me could improve myocardial cell damage and myocardial fibrosis in Streptozotocin (STZ)induced type 1 diabetic rats. The outcomes of this study indicated that $\mathrm{Me}$ inhibited the STZ-induced production of proinflammatory mediators, including TNF- $\alpha$ and IL-2, by suppressing the activation of NF- $\kappa \mathrm{B}$ and $\mathrm{Wnt} / \beta$-catenin/
GSK3 $\beta$ signaling pathways to alleviate cardiomyocyte hypertrophy and fibrosis (Liu et al., 2020).

Vincristine (VCR), a major vinca alkaloid, is commonly used in the treatments of various cancers, including lymphoma, leukemia, neuroblastoma, and so on (Said and Tsimberidou, 2014). Previous studies showed that VCR exerts cardioprotective effects on adult mouse myocytes or myocardial necrosis in rats. However, its efficacy on cardiac fibrosis treatment remains unclear (Panda et al., 2014). Therefore, Ge et al. conducted an experiment, in which ISOinjected adult male Sprague-Dawley (SD) rats were treated with VCR or vehicle. They found that VCR-treated rats showed further alleviation in the degree of myocardial fibrosis compared with the vehicle-treated rats, as demonstrated by the reduced heart/body weight ratio. The decreased colocalization among the nucleotide-binding domain, leucine-rich repeat, NLRP3, and ASC in VCR-treated rats could be observed. VCR could ameliorate cardiac fibrosis by down-regulating the expressions of caspase-1, IL-1 $\beta$, and IL-18 and then inhibiting the activation of the NLRP3 inflammasome directly (Ge et al., 2021). 6-Bromoindirubin- $3^{\prime}$-oxime (6BIO), an indirubin derivative, could attenuate cardiac fibrosis by increasing the anti-aging effects on aging heart. 6BIO promoted autophagy by decreasing the expression of p62 protein, increasing beclin1 level and LC3II/I ratio, inhibiting the ROS production, and eventually alleviating oxidative stress. In addition, 6BIO may suppress the GSK3 $\beta$ and mTOR pathway to delay the aging process of an aging heart (Guo et al., 2020).

Carvedilol is a nonselective third-generation $\beta$-adrenoceptor that has been used to treat myocardial injury and fibrosis induced by acute myocardium infarction (AMI) and diabetic cardiomyopathy (DCM) (Zhu et al., 2013). For example, in in vitro experiments, $\mathrm{Zhu}$ et al. reported that carvedilol suppressed the activation of ROS-induced $\operatorname{Smad} 3$ to reduce Colla1, Col3a1, and a-SMA expressions and increase miR-29b expression in a dose-dependent manner. These data suggested that carvedilol protected rat against AMI-induced myocardial fibrosis through the abrogation of Smad3 and the upregulation of miR-29b (Zhu et al., 2013). Okumura et al. investigated the effects of carvedilol on biventricular fibrosis and its functions against pulmonary arterial hypertension $(\mathrm{PAH})$ in a rat model. They reported that carvedilol decreased the concentration of collagen and the expression of TGF- $\beta 1$ and CTGF. Moreover, the hemodynamics and exercise endurance improved. Carvedilol could ameliorate biventricular fibrosis by blocking the TGF $\beta 1$ CTGF pathway (Okumura et al., 2015). A study conducted by Zheng et al. found that carvedilol could elevate cardiac function and further improve myocardial fibrosis against DCM rats by upregulating the AKT/XIAP antiapoptotic pathway and inhibiting myocardial inflammation (Zheng et al., 2017). Melatonin is an indoleamine produced by the pineal gland. It possesses a wide range of pharmacological effects on various tissue and organs and prevents myocardial fibrosis (Song et al., 2020). Several studies reported the protective activities of melatonin against cardiac fibrosis. In a study where diabetic mice were used as research objects, Zhou et al. reported a novel pathway to induce the occurrence of diabetic cardiomyopathy. This disease was 


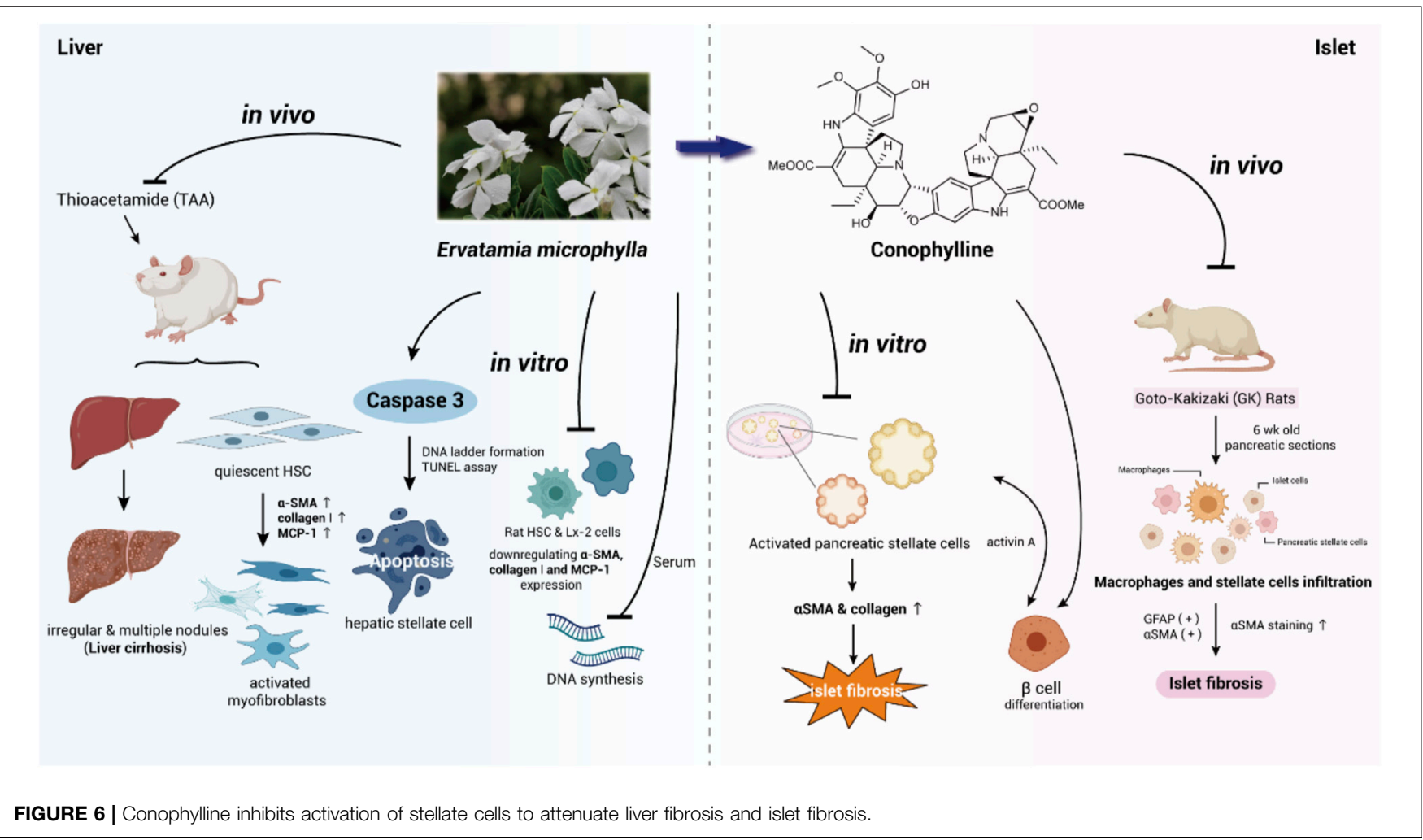

reportedly alleviated through the suppression of the activation of Syk/COX-1/SERCA axis by melatonin. Thus, it attenuated cardiac fibrosis and retained the vitality of cardiomyocytes (Zhou et al., 2018). In another study, Che et al. used diabetic mice and found that melatonin could significantly elevate cardiac dysfunction and exert antifibrotic effect by inhibiting the TGF- $\beta 1 /$ Smad pathway and activating the NLRP3 inflammasome (Che et al., 2020). Similarly, Jiang et al. showed that the administration of melatonin at $20 \mathrm{mg} / \mathrm{kg}$ for 4 weeks could relieve $\mathrm{PM}_{2.5}$-induced cardiac dysfunction and fibrosis in mice. Further research showed that melatonin could suppress mitochondrial oxidative injury and regulate the deacetylation of SOD2 mediated by SIRT3 to exert anti-fibrotic activities (Jiang et al., 2021). These findings suggested that melatonin might be a promising agent for treating myocardial fibrosis.

\section{Renal Fibrosis}

The main pathological characteristics of renal fibrosis are the activation and proliferation of fibroblasts, as well as the accumulation of extracellular matrix (ECM) deposited in the renal interstitium, which ultimately lead to the structural destruction and functional loss of renal tissue (Sun et al., 2016; Djudjaj and Boor, 2019). Renal fibrosis is a major pathological change and a common pathway of all chronic kidney diseases that progress to end-stage renal disease (Djudjaj and Boor, 2019). This condition in the kidney can be induced by multiple factors such as infection, injury, toxin and radiation, which cause the overproduction of free radicals and superoxide. These factors also stimulate the secretion of various cytokines, thus leading to the occurrence and development of renal fibrosis (Meng et al., 2014; Richter et al., 2015). TGF- $\beta 1$, the key diver of renal fibrosis, induces the transformation of renal fibroblasts into myofibroblasts and promotes the fibrosis of renal diseases (Gu et al., 2020). TNF- $\alpha$ can induce the inflammation process; it is an essential pro-inflammatory mediator in the promotion of renal fibrosis. A large number of studies have confirmed that natural indole alkaloids and their synthetic derivatives can improve renal fibrosis and delay the progression of kidney disease by regulating a variety of cytokine-mediated signal transduction pathways (Meng et al., 2015).

According to Xia et al., 3,3-Diindolylmethane (DIM), obtained from cruciferous vegetables, inhibits the development of interstitial collagen fibrosis, fibronectin and collagen-1 expressions, and local fibroblast activation; it also decreases the phosphorylation of Smad2/3 and upregulates the expression of Smad7, eventually alleviating kidney injury and renal fibrosis. The antifibrotic effect of DIM on unilateral ureteral obstruction (UUO)-induced mice model was closely associated with the inhibition of TGF- $\beta / \mathrm{Smad} 2 / 3$ signaling (Xia et al., 2018). Shima et al. screened 11 indole derivatives with anti-TNF- $\alpha$ effect from an indole derivative library; mitochonic acid 35 (MA-35) exhibited not only anti-TNF- $\alpha$ effect but also anti-TGF- $\beta 1$ effect by inhibiting the phosphorylation of Smad3 (Shima et al., 2017). MA-35 suppressed the phosphorylation of I $\kappa$ B kinase to exert anti-TNF- $\alpha$ activity in hepatic inflammation induced by LPS/GaIN in mice. In the unilateral ureter obstructed mouse model, MA-35 diminished renal 


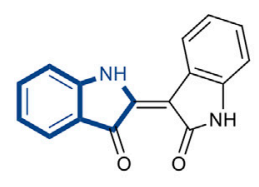

Indirubin (anti-pulmonary fibrosis)<smiles>C/C=C1\CN2CC[C@]1(CO)[C@H](C(=O)OC)c1[nH]c3ccccc3c1C2</smiles>

Vallesamine
(anti-pulmonary fibrosis)

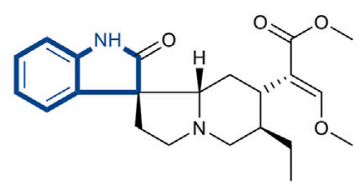

Isorhynchophylline (anti-pulmonary/myocardial fibrosis)<smiles>COC(=O)C1=C2Nc3c(O)cccc3[C@]23CCN2C[C@H](C(C)O)[C@H]1CC23</smiles>

19-epischolaricine (anti-pulmonary fibrosis)

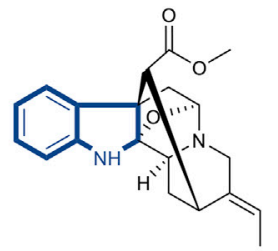

Picrinine (anti-pulmonary fibrosis)<smiles>O=Cc1ccc2cc[nH]c2c1</smiles>

Indole-6-carboxaldehyde (anti-pulmonary fibrosis)<smiles>COC(=O)C1=C(Nc2c(O)cccc2O)C2(C)CCN3CCC1(C(C)O)CC32</smiles>

Scholaricine (anti-pulmonary fibrosis)<smiles>CN1c2ccccc2C(=O)N2CCc3c([nH]c4ccccc34)[C@]21C</smiles>

Evodiamine (anti-pulmonary/myocardial/ liver fibrosis)

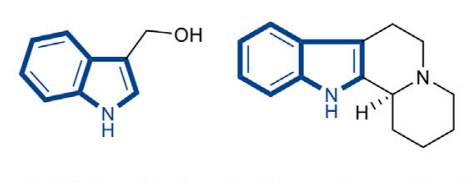<smiles>Brc1ccc2c3c([nH]c2c1)[C@H]1CCCCN1CC3</smiles><smiles>c1ccc2c(Cc3c[nH]c4ccccc34)c[nH]c2c1</smiles><smiles>O=c1c2ccccc2nc2n1CCc1c-2[nH]c2ccccc12</smiles>

Indole-3-carbinol Desbromoarborescidine $A$ (anti-pulmonary/ (anti-pulmonary fibrosis)

Arborescidine A (anti-pulmonary fibrosis)

3,3'-diindolymethane 3,3'-diindolymethane
(anti-myocardial/renal fibrosis)

Rutaecarpine (anti-myocardial fibrosis) liver fibrosis)

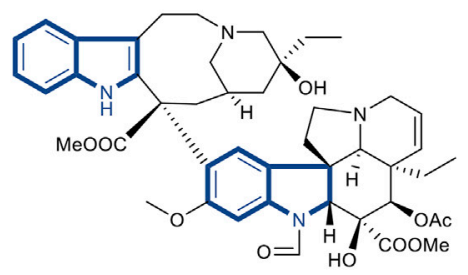

Vincristine (anti-myocardial fibrosis)<smiles>COC(=O)[C@H]1[C@@H](O)CC[C@H]2CN3CCc4c([nH]c5ccccc45)[C@H]3C[C@H]21</smiles>

Yohimbine (anti-renal fibrosis)

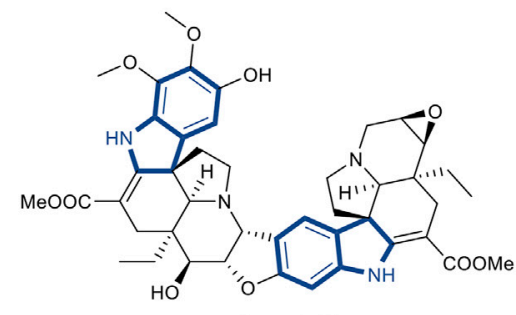

Conophylline (anti-liver/islet fibrosis)

Natural indole alkaloids<smiles>O=C1CC(n2ccc3cc(Cl)ccc32)C[C@@H]2[C@@H]3CCCN4CCC[C@@H](CN12)[C@H]34</smiles>

Compound $3 f$ (anti-pulmonary fibrosis)<smiles>CCOC(=O)C1=C[C@@]2(CC)CCCN3CCc4c(n1c1ccccc41)[C@@]32C</smiles>

Vinpocetine (anti-myocardial/liver fibrosis)<smiles>CN1C(=O)/C(=C2\C(=O)Nc3ccccc32)c2ccccc21</smiles>

Meisoindigo (anti-myocardial fibrosis)<smiles>O=C1Nc2cc(Br)ccc2/C1=C1\Nc2ccccc2\C1=N\O</smiles>

6-Bromoindirubin-3'-oxime (anti-myocardial fibrosis)<smiles>CN1CCN(c2ccc(C(=O)c3cc4cc([N+](=O)[O-])ccc4n3Cc3ccc(Br)cc3)cc2)CC1</smiles>

LG4<smiles>CCn1c(Br)c(CCN2C(=O)c3ccccc3C2=O)c2ccccc21</smiles>

Compound 8 (anti-liver fibrosis)<smiles>CN(C)CCCNc1nc(-c2[nH]c3ccccc3c2C=O)nc2ccccc12</smiles>

R17

(anti-liver fibrosis)

Semi-synthetic indole alkaloids

FIGURE 7 | (Continued) The chemical structures of anti-fibrotic indole alkaloids and indole derivatives.

inflammation and fibrosis by reducing the expression of inflammation factors and fibrotic genes, such as TNF- $\alpha$, iNOS, MCP-1, and IL-6. Furthermore, MA-35 might affect the recovery of epigenetic modifications and inhibit TNF- $\alpha$ / IKK and TGF- $\beta 1 / \mathrm{Smad} 3$ signaling to attenuate renal fibrosis (Figure 5, Shima et al., 2017). 


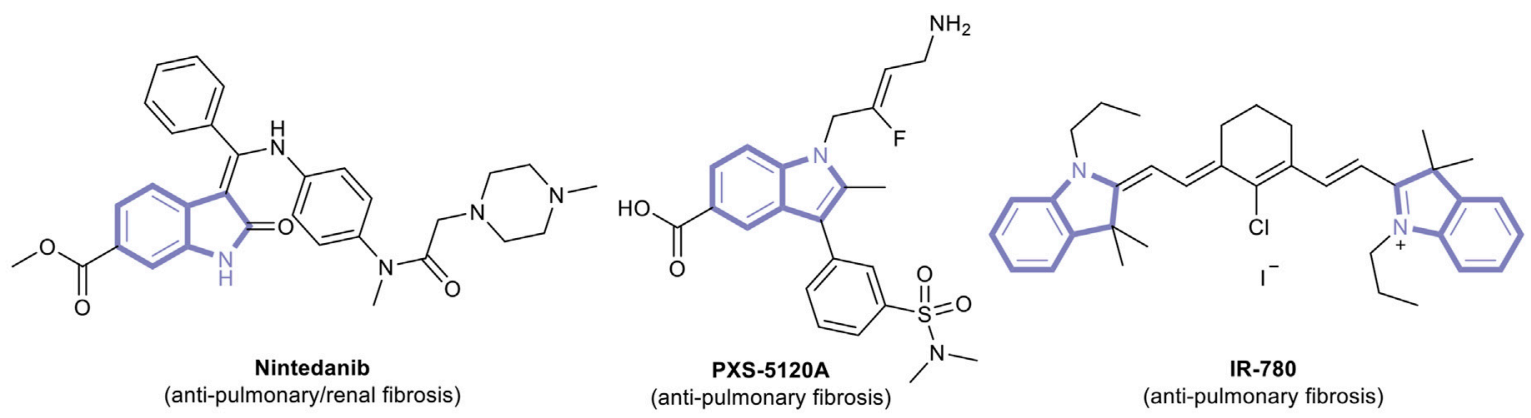<smiles>CCn1cc(NC(=O)Oc2cc(Cl)cc(Cl)c2)c2cc(-c3nc(CN4CCN(CCO)CC4)cs3)ccc21</smiles><smiles>COc1ccccc1OCCNC[C@H](O)COc1cccc2[nH]c3ccccc3c12</smiles><smiles>CC(=O)O/N=C1\C(=O)N(Cc2cc(Cl)ccc2Cl)c2ccc(Cl)cc21</smiles>

Type IV ATX inhibitors 66 (anti-pulmonary fibrosis)

Carvedilol (anti-myocardial/liver fibrosis)

LDN-57444

(anti-myocardial fibrosis)<smiles>COc1cc(COc2ccc3[nH]cc(CC(=O)O)c3c2)cc(OC)c1</smiles><smiles>COc1ccc(Cn2ccc3ccc(C(=O)NO)cc32)cc1</smiles>

PCI34051

(anti-renal fibrosis)<smiles>Cn1cc(C2=C(c3ccc(Cl)cc3Cl)C(=O)NC2=O)c2ccccc21</smiles>

(anti-renal fibrosis)

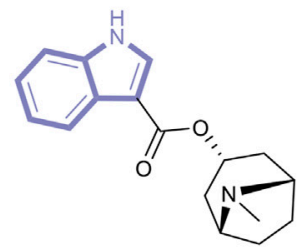

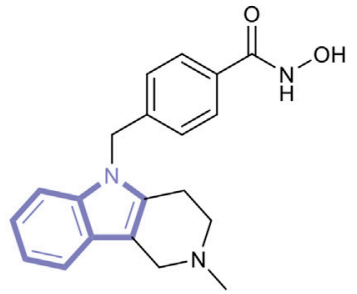

Tubastatin A (anti-renal fibrosis)
SB 216763

(anti-renal fibrosis)

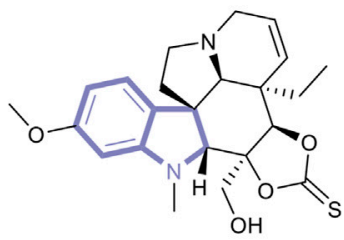

VD60

(anti-liver fibrosis) (anti-renal fibrosis)

Synthetic indole derivatives<smiles>COc1ccc2[nH]cc(CCNC(C)=O)c2c1</smiles>

Melatonin (anti-myocardial/renal/liver fibrosis)<smiles>O=Cc1c[nH]c2ccccc12</smiles>

Indole-3-carboxaldehyde (anti-liver fibrosis)<smiles>O=C(O)CCc1c[nH]c2ccccc12</smiles>

Indole-3-propionic acid (anti-liver fibrosis)<smiles>NCCc1c[nH]c2ccc(O)cc12</smiles>

Serotonin (anti-liver fibrosis)

Indole-contained metabolites

Yohimbine, an a2-adrenoceptor inhibitor from the dried bark extract of Corynante Yohimbe, showed renoprotective activities, according to Hayashi et al. (Hayashi et al., 2021). Results of experiments on a 5/6 nephrectomy-induced chronic kidney disease (CKD) rat model, showed that treatment with yohimbine significantly decreased urinary protein excretion 
TABLE 1 | Therapeutic activities of indole alkaloids and indole derivatives on organ fibrosis.

\begin{tabular}{|c|c|c|c|c|c|c|c|}
\hline Disease & Compound & Source & Study model & Stimulus & Dosage & $\begin{array}{l}\text { Activity/targets/ } \\
\text { pathway }\end{array}$ & References \\
\hline \multirow[t]{16}{*}{$\begin{array}{l}\text { Pulmonary } \\
\text { fibrosis }\end{array}$} & Indirubin & Muricidae & C57BL/6 mice & Bleomycin & $\begin{array}{l}12.5 \text { and } \\
25 \mathrm{mg} / \mathrm{kg}\end{array}$ & TGF- $\beta /$ Smad $\downarrow$ & Wang et al. (2020) \\
\hline & $\begin{array}{l}\text { Isorhynchophylline } \\
\text { (isorhy) }\end{array}$ & $\begin{array}{l}\text { Uncaria } \\
\text { rhynchophylla }\end{array}$ & Mice & $\mathrm{SiO}_{2}$ & $20 \mathrm{mg} / \mathrm{kg}$ & $\begin{array}{l}\text { TGF- } \beta 1, \text { TNF- } \alpha \text {, } \\
\text { IL-1 } \beta, I L-6 \downarrow \\
\text { collagen } \\
\text { deposition } \downarrow\end{array}$ & Qiu et al. (2020) \\
\hline & Picrinine & Alstonia scholaris & Mice & Bleomycin & $5 \mathrm{mg} / \mathrm{kg}$ & TGF- $\beta / M M P-1 \downarrow$ & Zhao et al. (2020); \\
\hline & Scholaricine & & & & $3 \mathrm{mg} / \mathrm{kg}$ & TGF- $\beta / M M P-1 \downarrow$ & Zhao et al. (2021) \\
\hline & Vallesamine & & & & $3 \mathrm{mg} / \mathrm{kg}$ & $\begin{array}{l}\text { IL-11, MMP-12, } \\
\text { TGF- } \beta \downarrow\end{array}$ & \\
\hline & 19-epischolaricine & & & & $1 \mathrm{mg} / \mathrm{kg}$ & $\begin{array}{l}\text { IL-11, MMP-12, } \\
\text { TGF- } \beta \downarrow\end{array}$ & \\
\hline & $\begin{array}{l}\text { Indole-6-carboxaldehyde } \\
\text { (I6CA) }\end{array}$ & $\begin{array}{l}\text { Sargassum } \\
\text { thunbergii }\end{array}$ & in vitro & $\mathrm{H}_{2} \mathrm{O}_{2}$ & $300 \mu \mathrm{M}$ & $\mathrm{Nrf2/HO}-1 \uparrow$ & Kim and Choi (2020) \\
\hline & Compound $3 f$ & Matrine derivative & MRC-5 cell lines & - & $\begin{array}{l}3.3 \pm 0.3 \mu \mathrm{M} \\
(\mathrm{IC} 50)\end{array}$ & $\begin{array}{l}\text { TGF- } \beta / \text { Smad } \\
\text { pathway } \downarrow\end{array}$ & Li et al. (2019b) \\
\hline & Evodiamine & Evodia rutaecarpa & BALB/c mice & Lipopolysaccharide & $10 \mathrm{mg} / \mathrm{kg}$ & Apelin $\uparrow$ & Ye et al. (2021) \\
\hline & Indole-3-carbinol (I3C) & $\begin{array}{l}\text { Brassica } \\
\text { vegetables }\end{array}$ & Rat pups & Hyperoxia-hypoxia & 100 mg/kg & $\begin{array}{l}\text { MMP-8, IL-6, NF- } \\
\kappa B \downarrow\end{array}$ & $\begin{array}{l}\text { Guzman-Navarro } \\
\text { et al. (2021) }\end{array}$ \\
\hline & Nintedanib & $\begin{array}{l}\text { Synthetic } \\
\text { compound }\end{array}$ & Human & COVID-19 & $\begin{array}{l}150 \text { mg } \\
\text { (twice daily) }\end{array}$ & $\begin{array}{l}\text { a-SMA, S100A4, } \\
\text { COL1, FN } \downarrow\end{array}$ & Umemura et al. (2021) \\
\hline & PXS-5120A & $\begin{array}{l}\text { Synthetic } \\
\text { compound }\end{array}$ & C57BL/6 mice & Bleomycin/ $\mathrm{CCl}_{4}$ & $20 \mathrm{mg} / \mathrm{kg}$ & $\mathrm{LOXL2/3 \downarrow}$ & Findlay et al. (2019) \\
\hline & $\begin{array}{l}\text { Desbromoarborescidine } \\
\text { A }\end{array}$ & $\begin{array}{l}\text { Dracontomelum } \\
\text { mangiferum }\end{array}$ & in vitro & - & $\begin{array}{l}82.5 \mu \mathrm{M} \\
\left(\mathrm{IC}_{50}\right)\end{array}$ & $\begin{array}{l}\text { showed cytotoxic } \\
\text { activity } \\
\text { towards human } \\
\text { lung } \\
\text { fibroblast cells }\end{array}$ & Santos et al. (2009) \\
\hline & Arborescidine A & $\begin{array}{l}\text { Pseudodistoma } \\
\text { arborescens }\end{array}$ & in vitro & - & $\begin{array}{l}71.6 \mu \mathrm{M} \\
\left(\mathrm{IC}_{50}\right)\end{array}$ & $\begin{array}{l}\text { showed cytotoxic } \\
\text { activity } \\
\text { towards human } \\
\text { lung } \\
\text { fibroblast cells }\end{array}$ & Santos et al. (2009) \\
\hline & Type IV ATX inhibitors 66 & $\begin{array}{l}\text { Synthetic } \\
\text { compound }\end{array}$ & C57Bl/6J mice & Bleomycin & $\begin{array}{l}20 \mathrm{or} \\
60 \mathrm{mg} / \mathrm{kg} \\
0.43 \mathrm{nM} \\
\left(\mathrm{IC}_{50}\right)\end{array}$ & $\begin{array}{l}\text { exerted a high } \\
\text { inhibition of the ex } \\
\text { vivo ATX activity }\end{array}$ & Lei et al. (2020) \\
\hline & IR-780 & $\begin{array}{l}\text { Synthetic } \\
\text { compound }\end{array}$ & $\begin{array}{l}\text { in vitro } \\
\text { C57BL/6 mice }\end{array}$ & Radiation & $0.4 \mathrm{mg} / \mathrm{kg}$ & $\begin{array}{l}\text { collagen I, } \alpha^{-} \\
\text {SMA } \downarrow\end{array}$ & Luo et al. (2021) \\
\hline \multirow[t]{9}{*}{$\begin{array}{l}\text { Myocardial } \\
\text { fibrosis }\end{array}$} & $\begin{array}{l}3,3^{\prime}- \\
\text { diindolymethane (DIM) }\end{array}$ & Cruciferous plants & in vivo & Adriamycin & $2.5 \mathrm{mg} / \mathrm{kg}$ & $\begin{array}{l}\text { BRCA1 } \uparrow \text { Nrf2 } \uparrow \\
\text { Collagen I } \downarrow \alpha- \\
\text { SMA } \downarrow\end{array}$ & Yao et al. (2013) \\
\hline & Rutaecarpine & Evodia rutaecarpa & Rats & Hypoxia & $\begin{array}{l}20 \mathrm{or} \\
40 \mathrm{mg} / \mathrm{kg}\end{array}$ & $\alpha-S M A, T G F-\beta 1 \downarrow$ & Li et al. (2016) \\
\hline & Evodiamine & Evodia rutaecarpa & Neonatal rats & TGF- $\beta 1$ & $\begin{array}{l}0.1,1,5 \\
10 \mu \mathrm{M}\end{array}$ & $\begin{array}{l}\text { TGF- } \beta 1 / \text { Smad } \downarrow \\
\alpha-S M A \text {, collagen-I/ } \\
\text { III, CTGF } \downarrow\end{array}$ & Wu et al. (2017b) \\
\hline & $\begin{array}{l}\text { Isorhynchophylline } \\
\text { (isorhy) }\end{array}$ & $\begin{array}{l}\text { Uncaria } \\
\text { rhynchophylla }\end{array}$ & C57BL/6 mice & Phenylephrine (PE) & $\begin{array}{l}0,5,10,25 \\
50 \mu \mathrm{M}\end{array}$ & $\begin{array}{l}\text { TGF- } \beta 1, \text { CTGF, } \\
\text { collagen } \mid / / I I \downarrow\end{array}$ & Zhang et al. (2020b) \\
\hline & Vinpocetine & $\begin{array}{l}\text { Vincamine } \\
\text { derivative }\end{array}$ & $\begin{array}{l}\text { C57/BL6 male } \\
\text { mice }\end{array}$ & Ang II & $5 \mathrm{mg} / \mathrm{kg}$ & $\begin{array}{l}\alpha-S M A, \text { collagen- } \\
1, E C M \downarrow\end{array}$ & Wu et al. (2017a) \\
\hline & Meisoindigo (Me) & Indirubin derivative & $\begin{array}{l}\text { Type } 1 \text { Diabetic } \\
\text { Rats }\end{array}$ & Streptozotocin (STZ) & $20 \mathrm{mg} / \mathrm{kg}$ & $\begin{array}{l}\text { NF- } \kappa B \downarrow \\
\text { Wnt } / \beta \text { catenin/ } \\
\text { GSK3 } \beta \downarrow\end{array}$ & Liu et al. (2020) \\
\hline & Vincristine (VCR) & $\begin{array}{l}\text { Catharanthus } \\
\text { roseus }\end{array}$ & $\begin{array}{l}\text { Sprague-Dawley } \\
\text { (SD) rats }\end{array}$ & Isoprotereno (ISO) & $\begin{array}{l}25 \text { and } \\
50 \mu \mathrm{g} / \mathrm{kg}\end{array}$ & $\begin{array}{l}\text { NLRP3 } \downarrow \text { caspase- } \\
1, I L-1 \beta, \| L-18 \downarrow\end{array}$ & Ge et al. (2021) \\
\hline & $\begin{array}{l}\text { 6-Bromoindirubin-3'- } \\
\text { oxime }(6 \mathrm{BIO})\end{array}$ & Indirubin derivative & C57BL/6J mice & - & $10 \mathrm{mg} / \mathrm{kg}$ & $\begin{array}{l}\text { p62, beclin- } 1 \text {, } \\
\text { LC3II/I, ROS } \downarrow \\
\text { GSK3 } \beta / \text { mTOR } \\
\text { pathway } \downarrow\end{array}$ & Guo et al. (2020) \\
\hline & Carvedilol & $\begin{array}{l}\text { Synthetic } \\
\text { compound }\end{array}$ & Rats & $\begin{array}{l}\text { ligating the left } \\
\text { anterior descending } \\
\text { coronary artery }\end{array}$ & $\begin{array}{l}1,5 \text { and } \\
10 \mathrm{mg} / \mathrm{kg}\end{array}$ & $\begin{array}{l}\text { Colla1, Col3a1, } a- \\
\text { SMA } \downarrow \text { miR-29b } \uparrow\end{array}$ & Zhu et al. (2013) \\
\hline
\end{tabular}

(Continued on following page) 
TABLE 1 | (Continued) Therapeutic activities of indole alkaloids and indole derivatives on organ fibrosis.

\begin{tabular}{|c|c|c|c|c|c|c|c|}
\hline Disease & Compound & Source & Study model & Stimulus & Dosage & $\begin{array}{c}\text { Activity/targets/ } \\
\text { pathway }\end{array}$ & References \\
\hline & & & Rats & Monocrotaline & $15 \mathrm{mg} / \mathrm{kg}$ & $\begin{array}{l}\text { TGF } \beta 1-C T G F \\
\text { signaling } \downarrow\end{array}$ & Okumura et al. (2015) \\
\hline & & & Rats & Streptozotocin (STZ) & $10 \mathrm{mg} / \mathrm{kg}$ & $\begin{array}{l}\text { AKT/XIAP } \uparrow \\
\text { caspase-3 } \downarrow\end{array}$ & Zheng et al. (2017) \\
\hline & Melatonin & Pineal gland & $\begin{array}{l}\text { Cardiac-specific } \\
\text { Syk knockout } \\
\text { mice }\end{array}$ & Streptozotocin (STZ) & $20 \mathrm{mg} / \mathrm{kg}$ & $\begin{array}{l}\text { Syk/COX-1/ } \\
\text { SERCA } \downarrow\end{array}$ & Zhou et al. (2018) \\
\hline & & & Mice & Streptozotocin (STZ) & $10 \mathrm{mg} / \mathrm{kg}$ & $\begin{array}{l}\text { TGF- } \beta 1 / \text { Smad } \downarrow \alpha- \\
\text { SMA } \downarrow \\
\text { NLRP3 } \downarrow\end{array}$ & Che et al. (2020) \\
\hline & & & $\mathrm{ApoE}^{-/-}$mice & $\mathrm{PM}_{2.5}$ & $20 \mathrm{mg} / \mathrm{kg}$ & $\begin{array}{l}\text { Collagen I/III } \downarrow \alpha^{-} \\
\text {SMA } \downarrow \text { SOD } 2 \uparrow\end{array}$ & Jiang et al. (2021) \\
\hline & LDN-57444 & $\begin{array}{l}\text { Synthetic } \\
\text { compound }\end{array}$ & $\begin{array}{l}\text { Spontaneously } \\
\text { hypertensive rats } \\
\text { (SHRs) }\end{array}$ & - & $20 \mu \mathrm{g} / \mathrm{kg}$ & TGF- $\beta / \mathrm{Smad} 2 / 3 \downarrow$ & Han et al. (2020) \\
\hline \multirow[t]{15}{*}{$\begin{array}{l}\text { Renal } \\
\text { fibrosis }\end{array}$} & $\begin{array}{l}3,3^{\prime}- \\
\text { Diindolylmethane (DIM) }\end{array}$ & Cruciferous plants & in vivo/in vitro & $\begin{array}{l}\text { Unilateral ureteral } \\
\text { obstruction (UUO) }\end{array}$ & 100 mg/kg & $\begin{array}{l}\text { TGF- } \beta / \text { Smad2/3 } \downarrow \\
\text { Collagen- } 1 \downarrow\end{array}$ & Xia et al. (2018) \\
\hline & $\begin{array}{l}\text { Mitochondrial acid } 35 \\
\text { (MA-35) }\end{array}$ & $\begin{array}{l}\text { Synthetic } \\
\text { compound }\end{array}$ & $\begin{array}{l}\text { LX-2 cells } \\
\text { C57BL/6 mice }\end{array}$ & LPS/D-GalN & $80 \mathrm{mg} / \mathrm{kg}$ & $\begin{array}{l}\text { TGF- } \beta 1, \text { TNF- } \alpha \text {, } \\
\text { iNOS } \downarrow \text { MCP- } 1, \text { IL- } \\
6 \downarrow\end{array}$ & Shima et al. (2017) \\
\hline & Yohimbine & Corynante Yohimbe & Rats & 5/6 nephrectomy & $\begin{array}{l}0.3 \mathrm{or} \\
3.0 \mathrm{mg} / \mathrm{L}\end{array}$ & $\begin{array}{l}\text { TGF- } \beta 1 \mathrm{mRNA} \text {, } \\
\text { collagen I } \downarrow\end{array}$ & Hayashi et al. (2021) \\
\hline & Nintedanib & $\begin{array}{l}\text { Synthetic } \\
\text { compound }\end{array}$ & $\begin{array}{l}\text { Male C57/Black } \\
\text { mice }\end{array}$ & $\begin{array}{l}\text { Unilateral ureteral } \\
\text { obstruction (UUO) }\end{array}$ & $50 \mathrm{mg} / \mathrm{kg}$ & $\begin{array}{l}\text { STAT3, NF-kB, } \\
\text { Smad3 } \downarrow \\
\text { PDGFR } \beta \text {, } \\
\text { VEGFR2 } \downarrow\end{array}$ & Liu et al. (2017) \\
\hline & & & $\begin{array}{l}\text { Precision-cut } \\
\text { kidney slices } \\
\text { (PCKS) }\end{array}$ & - & $0.1-10 \mu \mathrm{M}$ & $\begin{array}{l}\text { PDGFR, VEGFR } \downarrow \\
\text { Collagen- } 1 \downarrow\end{array}$ & Bigaeva et al. (2020) \\
\hline & PCI34051 & $\begin{array}{l}\text { Synthetic } \\
\text { compound }\end{array}$ & Murine & $\begin{array}{l}\text { Unilateral ureteral } \\
\text { obstruction (UUO) }\end{array}$ & $20 \mathrm{mg} / \mathrm{kg}$ & $\begin{array}{l}\alpha \text {-SMA, collagen } \downarrow \\
\text { TGF- } \beta 1 / \text { Smad3 } \downarrow \\
\text { STAT3 } \beta \text {-catenin } \downarrow\end{array}$ & Zhang et al. (2020c) \\
\hline & SB 216763 & $\begin{array}{l}\text { Synthetic } \\
\text { compound }\end{array}$ & Rats & Aldosterone (Aldo) & $1.5 \mathrm{mg} / \mathrm{kg}$ & $\begin{array}{l}\text { TNF- } \alpha, \text { IL-1 } \beta, \\
\text { MCP-1 } \downarrow \text { LC3-II } \uparrow \\
\text { p62 } \downarrow\end{array}$ & Zhang et al. (2018) \\
\hline & LG4 & $\begin{array}{l}\text { Indole-2- } \\
\text { Carboxamide } \\
\text { derivative }\end{array}$ & C57BL/6 mice & Streptozotocin (STZ) & $\begin{array}{l}5 \text { and } \\
10 \mathrm{mg} / \mathrm{kg}\end{array}$ & $\begin{array}{l}\text { TNF- } \alpha, I L-6, \downarrow \\
\text { MAPK/NF-кB } \downarrow\end{array}$ & Qian et al. (2021) \\
\hline & Tropisetron & $\begin{array}{l}\text { Synthetic } \\
\text { compound }\end{array}$ & Male Wistar rats & Streptozotocin (STZ) & $3 \mathrm{mg} / \mathrm{kg}$ & $\begin{array}{l}\text { TGF- } \beta 1, \text { p53 } \downarrow \\
\text { MMP-9, MMP-9 } \uparrow\end{array}$ & $\begin{array}{l}\text { Pourheydar et al. } \\
(2021)\end{array}$ \\
\hline & Melatonin & Pineal gland & Mice & Streptozotocin (STZ) & $20 \mathrm{mg} / \mathrm{kg}$ & AMPK/PGC1a $\uparrow$ & Li et al. (2019a) \\
\hline & & & C57BL/6N mice & Aristolochic acid (AA) & $20 \mathrm{mg} / \mathrm{kg}$ & TGF- $\beta 1 /$ Smad $\downarrow$ & Kim et al. (2019) \\
\hline & & & $\begin{array}{l}\text { NRK-49F cells } \\
\text { C57BL/6 mice }\end{array}$ & $\begin{array}{l}\text { TGF- } \beta 1 / \text { Unilateral } \\
\text { ureteral } \\
\text { obstruction (UUO) }\end{array}$ & $\begin{array}{l}20 \text { and } \\
50 \mathrm{mg} / \mathrm{kg}\end{array}$ & $\begin{array}{l}\text { STAT3, miR-21- } \\
\text { 5p } \downarrow \text { PTEN, } \\
\text { Spry1 } \uparrow\end{array}$ & Li et al. (2020a) \\
\hline & & & $\begin{array}{l}\mathrm{TH} 1 \text { cell BALB/c } \\
\text { mice }\end{array}$ & $\begin{array}{l}\text { P-cresol/ } 0.75 \% \\
\text { adenine }\end{array}$ & $1 \mu \mathrm{M}$ for $24 \mathrm{~h}$ & $\mathrm{miR}-4516 \uparrow \mathrm{ROS} \downarrow$ & Yoon et al. (2020) \\
\hline & & & C57BL/6 mice & $\begin{array}{l}\text { Cecal ligation } \\
\text { puncture (CLP) }\end{array}$ & $25 \mu \mathrm{g} / \mathrm{ml}$ & $\begin{array}{l}\text { IL- } 1 \alpha, I L-1 \beta, M c p- \\
1 \text { and TGF- } \beta 1 \text {, } \\
\operatorname{ROS} \downarrow\end{array}$ & Chen et al. (2021) \\
\hline & Tubastatin A & $\begin{array}{l}\text { Synthetic } \\
\text { compound }\end{array}$ & in vivo/in vitro & Angiotensin II (ANG) & $10 \mathrm{mg} / \mathrm{kg}$ & $\begin{array}{l}\text { Smad2/3, TGF- } \beta \text {, } \\
\text { CTGF, TNF- } \alpha \downarrow\end{array}$ & Choi et al. (2015) \\
\hline \multirow[t]{4}{*}{$\begin{array}{l}\text { Liver } \\
\text { fibrosis }\end{array}$} & Indole-3-carbinol (I3C) & $\begin{array}{l}\text { Brassica } \\
\text { vegetables }\end{array}$ & HSC-T6 cell & - & $\begin{array}{l}25,50, \text { and } \\
100 \mu \mathrm{M}\end{array}$ & $\begin{array}{l}\text { Bax/BCl-2 } \uparrow \text { CYLD } \\
\uparrow \text { RIP1 K63 de- } \\
\text { ubiquitination } \uparrow\end{array}$ & Li et al. (2017) \\
\hline & Compound 8 & $\begin{array}{l}\text { Phthalimide- } \\
\text { analog }\end{array}$ & in vitro/in vivo & $\mathrm{CCl}_{4}$ & $10 \mathrm{mg} / \mathrm{kg}$ & $\begin{array}{l}\text { Bcl-2 } \downarrow \text { Caspase-3 } \\
\uparrow \text { Improve the } \\
\text { fibrotic liver tissues } \\
\text { to normality }\end{array}$ & Zahran et al. (2018) \\
\hline & $\mathrm{R} 17$ & $\begin{array}{l}\text { Bouchardatine } \\
\text { derivative }\end{array}$ & C57BL/6J mice & high fat (HF) & $20 \mathrm{mg} / \mathrm{kg}$ & $\begin{array}{l}\text { TGF- } \beta \text {, collagen I, } \\
\text { MCP1 Smad3 } \downarrow\end{array}$ & Rao et al. (2019) \\
\hline & Conophylline (CnP) & $\begin{array}{l}\text { Ervatamia } \\
\text { microphylla }\end{array}$ & $\begin{array}{l}\text { BALB/c mice } L x- \\
2 \text { cells }\end{array}$ & Thioacetamide (TAA) & $4.09 \mathrm{mg} / \mathrm{ml}$ & $\begin{array}{l}\text { a-SMA, collagen- } 1 \\
\downarrow \text { caspase- } 3 \uparrow\end{array}$ & Kubo et al. (2014) \\
\hline
\end{tabular}

(Continued on following page) 
TABLE 1 | (Continued) Therapeutic activities of indole alkaloids and indole derivatives on organ fibrosis.

\begin{tabular}{|c|c|c|c|c|c|c|c|}
\hline Disease & Compound & Source & Study model & Stimulus & Dosage & $\begin{array}{l}\text { Activity/targets/ } \\
\text { pathway }\end{array}$ & References \\
\hline & Evodiamine & Evodia rutaecarpa & $\begin{array}{l}\text { Male Wistar rats } \\
\text { Hepatic stellate } \\
\text { cells }\end{array}$ & $\mathrm{CCl}_{4}$ & $\begin{array}{l}15 \text { and } \\
25 \mathrm{mg} / \mathrm{kg}\end{array}$ & $\begin{array}{l}\text { TGF- } \beta 1 / \text { Smad } \downarrow \\
\text { IL-6, TNF- } \alpha \text { and } \\
\text { collagen-I/III } \downarrow\end{array}$ & Yang et al. (2018) \\
\hline & \multirow[t]{2}{*}{ Vinpocetine } & \multirow[t]{2}{*}{$\begin{array}{l}\text { Vincamine } \\
\text { derivative }\end{array}$} & $\begin{array}{l}\text { Sprague-Dawley } \\
\text { rats }\end{array}$ & Thioacetamide (TAA) & $10-20 \mathrm{mg} / \mathrm{kg}$ & $\begin{array}{l}\text { Hydroxyproline, } \alpha \text { - } \\
\text { SMA } \downarrow \text { VEGF/KI- } \\
67 \downarrow\end{array}$ & Elnfarawy et al. (2021) \\
\hline & & & Male Wistar rats & $\begin{array}{l}\text { Diethylnitrosamine } \\
\text { (DEN) }\end{array}$ & 10 mg/kg & $\begin{array}{l}\text { TNF- } \alpha, \text { TLR4, } \\
\text { TIMP- } 1 \text { TGF- } \beta 1 \\
\text { and NF- } \mathrm{B} \downarrow \downarrow \\
\text { CREB } \uparrow\end{array}$ & Essam et al. (2019) \\
\hline & VD60 & $\begin{array}{l}\text { Synthetic } \\
\text { compound }\end{array}$ & in vitro/in vivo & $\mathrm{CCl}_{4}$ & $\begin{array}{l}10,15, \text { and } \\
25 \mathrm{mg} / \mathrm{kg}\end{array}$ & $\begin{array}{l}\alpha-S M A, T G F-\beta 1 \downarrow \\
\text { ROS, Akt, ERK, } \\
\text { Smad3 } \downarrow\end{array}$ & Wei et al. (2014) \\
\hline & Carvedilol & $\begin{array}{l}\text { Synthetic } \\
\text { compound }\end{array}$ & in vitro/in vivo & $\mathrm{CCl}_{4}$ & 10 mg/kg & $\begin{array}{l}\text { a-SMA, collagen I/ } \\
\text { III, ACE1 } \downarrow\end{array}$ & Wu et al. (2019) \\
\hline & $\begin{array}{l}\text { Indole-3-carboxaldehyde } \\
\text { (3-IAld) }\end{array}$ & $\begin{array}{l}\text { Lactobacillus } \\
\text { reuteri }\end{array}$ & C57BL/6 mice & $\begin{array}{l}3,5- \\
\text { diethoxycarbonyl- } \\
1,4- \\
\text { dihydrocollidine } \\
\text { (DDC) }\end{array}$ & 18 mg/kg & $\begin{array}{l}\alpha-S M A, T G F-\beta, I L- \\
9 \downarrow \text { AhR-IL-22 } \\
\text { axis } \uparrow\end{array}$ & D'Onofrio et al. (2021) \\
\hline & \multirow{5}{*}{$\begin{array}{l}\text { Indole-3-propionic } \\
\text { acid (IPA) } \\
\text { Melatonin }\end{array}$} & $\begin{array}{l}\text { Clostridium } \\
\text { Sporogenes }\end{array}$ & LX-2 cells & TGF- $\beta$ & $100 \mu \mathrm{M}$ & $\mathrm{COL} 1 \mathrm{~A} 2, \alpha-\mathrm{SMA} \downarrow$ & Sehgal et al. (2021) \\
\hline & & \multirow[t]{4}{*}{ Pineal gland } & Rats & $\mathrm{CCl}_{4}$ & $\begin{array}{l}2.5,5, \text { and } \\
10 \mathrm{mg} / \mathrm{kg}\end{array}$ & $\alpha-S M A, T G F-\beta \downarrow$ & Kang et al. (2016) \\
\hline & & & Mice & $\mathrm{CCl}_{4}$ & $\begin{array}{l}5 \text { or } \\
10 \mathrm{mg} / \mathrm{kg}\end{array}$ & $\begin{array}{l}\text { SphK1/S1P } \\
\text { pathway } \downarrow\end{array}$ & $\begin{array}{l}\text { Gonzalez-Fernandez } \\
\text { et al. (2017) }\end{array}$ \\
\hline & & & Rats & Thioacetamide (TAA) & $5 \mathrm{mg} / \mathrm{kg}$ & $\begin{array}{l}\text { thioredoxin-1 } \\
\text { mRNA } \\
\text { transcripts } \uparrow\end{array}$ & Lebda et al. (2018) \\
\hline & & & in vivo/in vitro & $\mathrm{CCl}_{4}$ & $\begin{array}{l}5 \mathrm{or} \\
10 \mathrm{mg} / \mathrm{kg}\end{array}$ & $\begin{array}{l}\alpha-S M A, \text { collagen-1 } \\
\downarrow \text { PPAR- } \alpha \uparrow\end{array}$ & $\begin{array}{l}\text { Mortezaee et al. } \\
\text { (2017) }\end{array}$ \\
\hline & Serotonin & Metabolite & C57BL/6 mice & Concanavalin A & - & $\begin{array}{l}\text { TGF- } \beta 1 / \text { Smads } \downarrow \\
\text { IL-6, IFN- } \gamma \text {, TNF- } \alpha \text {, } \\
\text { TGF- } \beta 1 \downarrow\end{array}$ & Pang et al. (2021) \\
\hline Islet fibrosis & Conophylline (CnP) & $\begin{array}{l}\text { Tabernaemontana } \\
\text { divaricata }\end{array}$ & $\begin{array}{l}\text { Male Goto- } \\
\text { Kakizaki (GK) } \\
\text { rats }\end{array}$ & - & $0.9 \mu \mathrm{g} / \mathrm{g}$ & $\begin{array}{l}\text { Collagen, } \alpha \text {-SMA, } \\
\text { MCP- } 1 \downarrow \text { PSCs } \downarrow\end{array}$ & Saito et al. (2012) \\
\hline
\end{tabular}

and noradrenaline content of renal venous plasma compared with hydralazine-treated animals. Yohimbine treatment could suppress the mRNA expression levels of TGF- $\beta$, collagen III, and fibronectin, thus inhibiting renal fibrosis. Yohimbine has potential as a therapeutic drug for CKD (Hayashi et al., 2021). Nintedanib, a small-molecule tyrosine kinase inhibitor, is an FDA-approved drug that is used to treat idiopathic pulmonary fibrosis. Recently, it was reported that nintedanib has a moderating effect on renal fibrosis. Liu et al. stated that in the UUO-induced mouse model, the administration of $50 \mathrm{mg} / \mathrm{kg}$ nintedanib greatly reduced the activation of renal interstitial fibroblasts and ameliorated renal fibrosis. Additionally, nintedanib suppressed PDGFR $\beta$, FGFR1, FGFR2, and VEGFR2 phosphorylation and blocked the expressions of STAT3, NF- $\kappa$ B, and Smad3 to diminish macrophage infiltration, thereby ultimately exerting anti-renal fibrosis effects (Liu et al., 2017). In another study, Bigaeva et al. used precision-cut kidney slices (PCKS) as an experimental model to study the effect of nintedanib on renal fibrosis. The study showed that nintedanib could inhibit the phosphorylation of PDGFR and VEGFR, suppress cell proliferation, and decrease the content of collagen-I and the expression of fibrosis-related genes, thereby protecting against the occurrence of fibrosis in both murine and human PCKS. However, it could not reverse fibrosis (Bigaeva et al., 2020).

Zhang et al. reported a novel target for treating renal fibrosis, namely, histone deacetylase 8 (HDAC8), which is expressed in renal tubular epithelial cells. In UUO-induced renal fibrosis mice model, the HDAC8 inhibitor, PCI34051, inhibited Smad3, STAT3, $\beta$-catenin and Snail expressions and activated BMP7 and Klotho renal protective protein expressions. In addition, fibrotic markers, including a-SMA, collagen 1, and fibronectin, could be inhibited, thereby alleviating the occurrence of renal fibrosis (Zhang et al., 2020c). SB-216763, as a GSK-3 $\beta$ inhibitor, could reverse the hypertrophy and dysfunction of the heart and kidneys in aldosterone (Aldo)-induced rats by suppressing the expression of inflammatory factors including TNF-a, IL- $1 \beta$, and MCP-1. SB-216763 triggered the activation of autophagy by 
upregulating the LC3-II protein levels and promoting p62 protein degradation in cardiac and renal tissues, which played an important role in ameliorating perivascular fibrosis, and renal injury (Zhang et al., 2018).

A synthetic indole-contained compound, LG4, has been improved to possess anti-inflammatory activities both in vitro and in vivo (Liu et al., 2019b). Qian et al. found that LG4 has renal protective effects on diabetic kidney disease (DKD) in T1MD mice. Moreover, it could significantly ameliorate glomerulosclerosis and fibrosis induced by hyperglycemia in TIDM mice without affecting the body weight and blood glucose levels. These effects were manifested in the decreased expressions of COL- 4 and TGF- $\beta$. Additionally, in vivo and in vitro data showed that LG4 could improve antiinflammatory effects by suppressing the phosphorylation of JNK and ERK and by activating NF- $\kappa B$ signaling. However, the renal protective activity of $\mathrm{DKD}$ in type 2 diabetic mice needs further study (Qian et al., 2021). Tropisetron, a synthetic indole drug, has been used to inhibit chemotherapy-induced vomiting in clinic. Previous research showed that tropisetron has multiple pharmacological properties including antiinflammatory, anti-diabetic, and anti-fibrotic (Barzegar-Fallah et al., 2015). In this study, Pourheydar et al. investigated the anti-renal fibrosis effects of tropisetron against diabetic nephropathy rat model. The results showed that tropisetron could ameliorate kidney function and attenuate renal fibrosis by inhibiting the expressions of TGF- $\beta 1$ and p53 proteins, as well increasing the level of extracellular matrix metalloproteinases, including MMP-9 and MMP-2 (Pourheydar et al., 2021).

Melatonin is a pineal hormone with strong anti-oxidant and anti-inflammatory effects. Recently, several studies showed that melatonin has a certain protective effect on kidney injury and anti-renal fibrosis, especially for the fibrosis of diabetic mice, which has been widely studied. In animal models of renal fibrosis caused by hyperglycemia, Li et al. reported that melatonin exerted anti-renal fibrosis activities, as shown by the restoration of mitochondrial function and the activation of the AMPK/PGC1 $\alpha$ pathway (Li et al., 2019a). For aristolochic acid (AA)-induced nephropathy (AAN) mice model, the contents of blood urea nitrogen and creatinine decreased, and renal tubules dilated, but treatment with melatonin could reverse these indexes. Melatonin alleviated tubulointerstitial fibrosis by inhibiting the TGF- $\beta /$ Smad pathway (Kim et al., 2019). Additionally, according to $\mathrm{Li}$ et al., melatonin was used to ameliorate TGF- $\beta 1$-induced renal fibroblast to myofibroblast transdifferentiation (FMT) and UUO-induced renal fibrosis by decreasing the levels of a-SMA, collagen-I, fibronectin, and miR-21-5p and the phosphorylation of STAT3 and by increasing the expressions of Spryl and PTEN (Li et al., 2020a). Toon et al. found that melatonin is a promising drug for the treatment of a chronic kidney disease (CKD) patient with renal fibrosis; melatonin could recover mitochondrial function by upregulating the miR4516 expression to decrease ROS formation (Yoon et al., 2020). In response to acute kidney injury induced by sepsis, melatonin could reduce the oxidative stress response and ROS accumulation of ROS in vitro and attenuate the inflammatory response by downregulating the mRNA expressions of IL-1 $\alpha$, IL- $1 \beta, \mathrm{Mcp}-1$ and TGF- $\beta 1$, thereby proving the therapeutic effect of melatonin on acute kidney injury (Chen et al., 2021).

\section{Liver Fibrosis}

Liver fibrosis (LF) is a self-injury repair reaction caused by chronic liver injury, including hepatitis virus infections, metabolic diseases, non-alcoholic steatohepatitis and immune liver injury. If liver fibrosis is not effectively controlled, then it will eventually develop into cirrhosis or lead to life-threatening liver cancer (Parola and Pinzani, 2019; Zhangdi et al., 2019). The formation of hepatic fibrosis includes the activation and transformation of hepatic stellate cells, collagen deposition, and remodeling of extracellular matrix proteins (Campana and Iredale, 2017). Hepatic fibrosis is reversible. Several indole alkaloid compounds regulate the TGF- $\beta /$ Smad, NF- $\kappa B$, MAPK, and other signaling pathways to inhibit oxidative stress and inflammatory response and then reduce liver injury to alleviate hepatic fibrosis. Hepatic stellate cells (HSCs) are the main cell sources of matrix components. They play an essential role in the occurrence and development of liver fibrosis (Novo et al., 2014; Higashi et al., 2017). Induction of HSC apoptosis is a potential way to reverse liver fibrosis (Elsharkawy et al., 2005). According to Li et al., indole-3-carbinol (I3C), a natural compound obtained from Brassica vegetables, could induce HSC apoptosis to attenuate hepatic fibrosis by increasing deubiquitinase cylindromatosis (CYLD) level and decreasing K63ubiquitination of RIP1. These effects were closely related to the upregulation of $\mathrm{Bax} / \mathrm{Bcl}-2$ ratio and caspase- 8 , as well as the inhibition of the NF- $\mathrm{BB}$ pathway (Li et al., 2017). Zahran et al. demonstrated that phthalimide-indole analogs, including compound 8, effectively improved the anti-liver fibrotic activity by downregulating the $\mathrm{Bcl}-2$ protein and upregulating caspase- 3 to promote apoptosis. Compound 8 also possessed strong anticancer effect on HepG2, MCF-7, A549 and other cell lines. According to histopathological studies, compound 8 treatment could restore fibrotic liver tissue to normal (Zahran et al., 2018).

Conophylline ( $\mathrm{CnP})$, an indole alkaloid extracted from tropical plant Ervatamia microphylla, reportedly attenuates liver fibrosis. In rat HSCs and $\mathrm{Lx}-2$ cells, CnP decreased the $\alpha$ SMA and collagen-1 expressions and DNA synthesis, as well as upregulated the caspase- 3 to induce apoptosis. In thioacetamideinduced rats, the liver surface of rats was not smooth, and many nodules were present. $\mathrm{CnP}$ treatment reversed these phenomena, and the concentration of collagen decreased. CnP could be a promising compound for the treatment of hepatic fibrosis (Figure 6; Kubo et al., 2014). In Yang et al.'s study, evodiamine ameliorated liver fibrosis by inhibiting the TGF$\beta 1 /$ Smad pathway. Evodiamine decreased the contents of IL- 6 , TNF- $\alpha$, and collagen-I/III. The level of TGF- $\beta 1$, phosphorylation of Smad 2/3, and a-SMA were blocked by evodiamine in $\mathrm{CCl}_{4}{ }^{-}$ induced liver fibrosis rat model. Meanwhile, evodiamine suppressed cell proliferation, hydroxyproline production, and collagen metabolism in HSCs in a dose-dependent manner (Yang et al., 2018). These data proved that evodiamine might 
be a potential therapeutic drug for treating fibrotic liver diseases (Li et al., 2020b).

Inflammatory cytokines play an important role in initiating the activation and regulation of liver fibrosis. Elnfarawy et al. tested the anti-fibrotic activity of a synthetic vinca alkaloid vincamine derivative, namely, vinpocetine against thioacetamide-induced liver fibrosis rat model. By downregulating the hydroxyproline and a-SMA level, the liver oxidative stress and histopathological damage were significantly improved. This group discovered that vinpocetine could inhibit angiogenesis and proliferation by reducing VEGF/KI-67 expression (Elnfarawy et al., 2021). Cyclic adenosine monophosphate (cAMP) can inhibit the proliferation of fibroblasts and the synthesis of ECM protein. The activity of cAMP is regulated by phosphodiestrases (PDEs) to some extent (Insel et al., 2012). To explore the mechanism of a PDE inhibitor in liver fibrosis by using vinpocetine as the PDE-1 inhibitor, Essam et al. studied its therapeutic effect in diethylnitrosamine (DEN)-induced liver fibrosis. Their results showed that TNF- $\alpha$, TLR4, and TIMP-1 expressions decreased and CREB protein expression increased remarkably. Furthermore, the concentrations of hydroxyproline, TGF- $\beta 1$ and NF- $\kappa \mathrm{B}$ were significantly inhibited (Essam et al., 2019). Additionally, Alhusseiny et al. reported that vinpocetine can also be used as a novel adjuvant of praziquantel to attenuate liver fibrosis of schistosoma (Alhusseiny et al., 2018).

VD60 is a novel indol-contained skeleton $\mathrm{CB}_{1}$ inhibitor. Wei et al. investigated the anti-hepatic fibrosis effects of VD60 in vitro and in vivo (Wei et al., 2014). In the human HSC line LX-2 and rat HSCs, VD60 inhibited the expression of $a_{2}$ (I) procollagen mRNA, the production of ROS, and the phosphorylation of Akt, ERK, and Smad3, thereby exerting a remarkable anti-proliferation activity. In $\mathrm{CCl}_{4}$-induced liver fibrosis mouse model, the VD60-treated group showed that a-SMA expression, TGF- $\beta$, and fibronectin mRNA levels, the fibrotic area, and hepatic hydroxyproline (HYP) deposition all decreased compared with the vehicle group, suggesting that VD60 could effectively ameliorate liver fibrosis. They measured VD60 concentration in the plasma and brain, and the results showed that the content of VD60 in blood was much higher than that in brain. This finding indicated that VD60 could not penetrate the blood-brain barrier and played an anti-fibrosis role as a peripheral CB1 antagonist (Wei et al., 2014). Wu et al. discovered that carvedilol retarded the cell cycle at the G0/G1 phase and decreased the $\alpha$-SMA expression and collagen I/III deposition to inhibit the proliferation of HSC induced by Ang II, thereby ameliorating hepatic fibrosis. Additionally, carvedilol promoted HSC apoptosis by down-regulating Bcl-2 protein expression and attenuated liver fibrosis (Wu et al., 2019). Indole-3-carboxaldehyde (3-IAld), an indole metabolite, was produced from commensal Lactobacillus reuteri. 3-IAld promoted the recovery of intestinal barrier function and played an important role in the treatment of liver and gastrointestinal diseases. In the DCC-induced PSC murine model, Onofrio et al. found that 3-IAld could reduce the formation of liver fibrosis by inhibiting the expressions of TGF- $\beta 1$ and IL-9. It maintained intestinal mucosa homeostasis by regulating intestinal flora and activating the microbiota aryl hydrocarbon receptor (AhR)-IL-22 axis, thus preventing and ameliorating liver inflammation and fibrosis (D'Onofrio et al., 2021). As an indoleamine compound, melatonin affects the activity of hepatic stellate cells and has an inhibitory effect on some pro-inflammatory factors, such as TNF- $\alpha$, IL- $1 \beta$, IL- 6 , and TGF- $\beta 1$. In some experimental animal models of hepatic fibrosis, melatonin regulates oxidative stress, inflammatory response, and apoptosis to prevent and treat liver injury and liver fibrosis caused by various factors (Kang et al., 2016; Gonzalez-Fernandez et al., 2017; Mortezaee et al., 2017; Lebda et al., 2018).

\section{Islet Fibrosis}

Islet fibrosis occurs in patients with type 2 diabetes mellitus (T2DM). It may interfere with the metabolism of pancreatic $\beta$ cells, thereby affecting insulin secretion, destroying the normal structure of islet, and promoting the occurrence of T2DM (Katsuda et al., 2014). The pathogenesis of islet fibrosis may be related to the activation of pancreatic stellate cells (PSCs). Under the stimulation of chronic pancreatitis and pancreatic cancer, PSCs are activated to express $\alpha$-smooth muscle actin ( $\alpha$-SMA), secrete collagen, and synthesize amounts of extracellular matrix proteins, resulting in islet fibrosis (Lee et al., 2017; Xue et al., 2018). Goto-Kakizaki (GK) rats are among the most characteristic animal models of spontaneous type 2 diabetes mellitus, which is characterized by a decrease in the number of pancreatic $\beta$-cells and fibrosis formation. The metabolism and diabetes indicators of $\mathrm{GK}$ rats are very similar to human T2DM. Thus, they can be used for the study of islet fibrosis (Homo-Delarche et al., 2006).

In a study by Saito et al., conophylline (CnP), a natural indole alkaloid, obtained from the leaves of E. microphylla, reportedly exerts anti-islet fibrosis activity against Goto-Kakizaki rat model (Saito et al., 2012). CnP could suppress the activation of stellate cells and decrease the collagen-I production. In 6-week-old GK rat pancreatic sections, the CD68-positive macrophages and GFAPand a-SMA-positive stellate cells infiltrated into islets, and subsequently, macrophages and $\alpha$-SMA stained stellate cells increased. This finding suggested that stellate cells were involved in islet fibrosis in GK rats. In in vivo experiments, after treating GK rats with $0.9 \mu \mathrm{g} / \mathrm{g}$ dose of $\mathrm{CnP}$ for 4 weeks, the invasion of PSCs and macrophages decreased significantly, and the concentrations of insulin elevated greatly. All the abovementioned data proved that $\mathrm{CnP}$ is a promising compound for ameliorating islet fibrosis both in vitro and in vivo (Figure 6; Saito et al., 2012). In another study, conophylline inhibited the activity and proliferation of cancerassociated fibroblasts (CAF) and reduced the desmoplasia of cancer tissue. It suppressed tumor proliferation when combined with antitumor drug, gemcitabine (Ishii et al., 2019). A brief illustration of all indole alkaloids is presented in Figure 7 and Table 1.

\section{CONCLUSION AND OUTLOOKS}

The current review summarizes the recent advancements in the use of indole alkaloids and indole derivatives to treat organ fibrosis. Multiple organ damage can trigger complex cellular and 
molecular cascades that eventually lead to fibrosis. Some natural indole alkaloids show perfect anti-inflammatory, antiviral, and antibacterial effects. Thus, their medicinal value is beyond doubt and deserves more attention. In this review, through the analysis of the related targets and pathways of pulmonary, myocardial, renal, liver, and islet fibrosis, small molecules containing indole skeletons regulated TGF- $\beta 1 / \mathrm{Smad}, \mathrm{NF}-\kappa \mathrm{B}, \mathrm{Wnt} / \beta$-catenin, $\mathrm{Nrf} 2 / \mathrm{HO}-1$, and other pathways to inhibit different types of fibrosis. Current reports showed that although many research groups have demonstrated the potential of indole alkaloids in the treatment of organ fibrosis through in vivo and in vitro trials, only a few clinical trials have been conducted. Therefore, further preclinical and clinical trials are needed to evaluate the efficacy of these natural and synthetic indole alkaloids and to determine whether they can be successfully used in the clinical treatment of human organ fibrosis. In the future, we hope that more indole alkaloids can be found and synthesized. Clinical studies can be carried out on these indole alkaloids. Their structures can be modified and transformed appropriately to improve the efficacy. Currently, one of the major obstacles of translating novel antifibrotic drugs to the clinic is due to the intricacies of drug delivery. We may be able to load such small molecules on nanoparticles to improve drug stability and targeting, and reduce unwanted cellular uptake. Treatment using nanoparticle systems is a promising tool for the therapy of chronic inflammatory diseases, which provides new perspectives and ideas for the treatment of fibrosis. These future prospects can

\section{REFERENCES}

Abdelfatah, S. A. A., and Efferth, T. (2015). Cytotoxicity of the Indole Alkaloid Reserpine from Rauwolfia Serpentina against Drug-Resistant Tumor Cells. Phytomedicine 22 (2), 308-318. doi:10.1016/j.phymed.2015.01.002

Alhusseiny, S. M., El-Beshbishi, S. N., Abu Hashim, M. M., El-Nemr, H. E. E., and Handoussa, A. E. (2018). Effectiveness of Vinpocetine and Isosorbide-5-Mononitrate on Experimental Schistosomiasis Mansoni: Biochemical and Immunohistochemical Study. Acta Trop. 186, 16-23. doi:10.1016/j.actatropica.2018.06.010

Barzegar-Fallah, A., Alimoradi, H., Asadi, F., Dehpour, A. R., Asgari, M., and Shafiei, M. (2015). Tropisetron Ameliorates Early Diabetic Nephropathy in Streptozotocin-Induced Diabetic Rats. Clin. Exp. Pharmacol. Physiol. 42 (4), 361-368. doi:10.1111/1440-1681.12373

Beuselinck, B., Job, S., Becht, E., Karadimou, A., Verkarre, V., Couchy, G., et al. (2015). Molecular Subtypes of Clear Cell Renal Cell Carcinoma Are Associated with Sunitinib Response in the Metastatic Setting. Clin. Cancer Res. 21 (6), 1329-1339. doi:10.1158/1078-0432.Ccr-14-1128

Bigaeva, E., Stribos, E. G. D., Mutsaers, H. A. M., Piersma, B., Leliveld, A. M., de Jong, I. J., et al. (2020). Inhibition of Tyrosine Kinase Receptor Signaling Attenuates Fibrogenesis in an Ex Vivo Model of Human Renal Fibrosis. Am. J. Physiol. Ren. Physiol 318 (1), F117-f134. doi:10.1152/ajprenal.00108.2019

Campana, L., and Iredale, J. P. (2017). Regression of Liver Fibrosis. Semin. Liver Dis. 37 (1), 1-10. doi:10.1055/s-0036-1597816

Chadha, N., and Silakari, O. (2017). Indoles as Therapeutics of Interest in Medicinal Chemistry: Bird's Eye View. Eur. J. Med. Chem. 134, 159-184. doi:10.1016/j.ejmech.2017.04.003

Chanda, D., Otoupalova, E., Smith, S. R., Volckaert, T., De Langhe, S. P., and Thannickal, V. J. (2019). Developmental Pathways in the Pathogenesis of Lung Fibrosis. Mol. Aspects Med. 65, 56-69. doi:10.1016/j.mam.2018.08.004

Che, H., Wang, Y., Li, H., Li, Y., Sahil, A., Lv, J., et al. (2020). Melatonin Alleviates Cardiac Fibrosis via Inhibiting lncRNA MALAT1/miR-141-Mediated NLRP3 Inflammasome and TGF- $\beta 1 /$ Smads Signaling in Diabetic Cardiomyopathy. FASEB J. 34 (4), 5282-5298. doi:10.1096/fj.201902692R lead to certain strategies for the development of new targeted fibrosis drugs.

\section{DATA AVAILABILITY STATEMENT}

The original contributions presented in the study are included in the article/Supplementary Material, further inquiries can be directed to the corresponding authors.

\section{AUTHOR CONTRIBUTIONS}

WH and GZ contributed to the conception and design of the study. RQ and QZ organized the database, performed the statistical analysis, and wrote the first draft of the manuscript. $\mathrm{BH}, \mathrm{H}-\mathrm{PZ}$, and $\mathrm{CP}$ contributed to the manuscript revision. All authors read and approved the submitted version.

\section{FUNDING}

We are grateful for financial support from National Natural Science Foundation of China (82073997, 22001024, 82073998), Sichuan Science and Technology Program (2021YFS0044, 2022CXRC0077, 2021YJ0402) and Xinglin Scholar Research Promotion Project of Chengdu University of TCM.

Chen, L., Yang, T., Lu, D. W., Zhao, H., Feng, Y. L., Chen, H., et al. (2018). Central Role of Dysregulation of TGF- $\beta /$ Smad in CKD Progression and Potential Targets of its Treatment. Biomed. Pharmacother. 101, 670-681. doi:10.1016/ j.biopha.2018.02.090

Chen, L., Han, Z., Shi, Z., Liu, C., and Lu, Q. (2021). Melatonin Alleviates Renal Injury in Mouse Model of Sepsis. Front. Pharmacol. 12, 697643. doi:10.3389/ fphar.2021.697643

Choi, S. Y., Ryu, Y., Kee, H. J., Cho, S. N., Kim, G. R., Cho, J. Y., et al. (2015). Tubastatin A Suppresses Renal Fibrosis via Regulation of Epigenetic Histone Modification and Smad3-Dependent Fibrotic Genes. Vascul Pharmacol. 72, 130-140. doi:10.1016/j.vph.2015.04.006

Djudjaj, S., and Boor, P. (2019). Cellular and Molecular Mechanisms of Kidney Fibrosis. Mol. Aspects Med. 65, 16-36. doi:10.1016/j.mam.2018.06.002

D'Onofrio, F., Renga, G., Puccetti, M., Pariano, M., Bellet, M. M., Santarelli, I., et al. (2021). Indole-3-Carboxaldehyde Restores Gut Mucosal Integrity and Protects from Liver Fibrosis in Murine Sclerosing Cholangitis. Cells 10 (7), 1622. doi:10.3390/cells10071622

Elnfarawy, A. A., Nashy, A. E., Abozaid, A. M., Komber, I. F., Elweshahy, R. H., and Abdelrahman, R. S. (2021). Vinpocetine Attenuates Thioacetamide-Induced Liver Fibrosis in Rats. Hum. Exp. Toxicol. 40 (2), 355-368. doi:10.1177/0960327120947453

Elsharkawy, A. M., Oakley, F., and Mann, D. A. (2005). The Role and Regulation of Hepatic Stellate Cell Apoptosis in Reversal of Liver Fibrosis. Apoptosis 10 (5), 927-939. doi:10.1007/s10495-005-1055-4

Essam, R. M., Ahmed, L. A., Abdelsalam, R. M., and El-Khatib, A. S. (2019). Phosphodiestrase-1 and 4 Inhibitors Ameliorate Liver Fibrosis in Rats: Modulation of cAMP/CREB/TLR4 Inflammatory and Fibrogenic Pathways. Life Sci. 222, 245-254. doi:10.1016/j.lfs.2019.03.014

Findlay, A. D., Foot, J. S., Buson, A., Deodhar, M., Jarnicki, A. G., Hansbro, P. M., et al. (2019). Identification and Optimization of Mechanism-Based Fluoroallylamine Inhibitors of Lysyl Oxidase-Like 2/3. J. Med. Chem. 62 (21), 9874-9889. doi:10.1021/acs.jmedchem.9b01283

Foglia, B., Cannito, S., Bocca, C., Parola, M., and Novo, E. (2019). ERK Pathway in Activated, Myofibroblast-Like, Hepatic Stellate Cells: A Critical Signaling Crossroad Sustaining Liver Fibrosis. Int. J. Mol. Sci. 20 (11), 2700. doi:10. 3390/ijms20112700 
Gaboriaud-Kolar, N., Vougogiannopoulou, K., and Skaltsounis, A. L. (2015). Indirubin Derivatives: A Patent Review (2010 - Present). Expert Opin. Ther. Pat 25 (5), 583-593. doi:10.1517/13543776.2015.1019865

Ge, C., Cheng, Y., Fan, Y., and He, Y. (2021). Vincristine Attenuates Cardiac Fibrosis through the Inhibition of NLRP3 Inflammasome Activation. Clin. Sci. (Lond) 135 (11), 1409-1426. doi:10.1042/cs20210189

George, P. M., Wells, A. U., and Jenkins, R. G. (2020). Pulmonary Fibrosis and COVID-19: The Potential Role for Antifibrotic Therapy. Lancet Respir. Med. 8 (8), 807-815. doi:10.1016/s2213-2600(20)30225-3

González-Fernández, B., Sánchez, D. I., Crespo, I., San-Miguel, B., Álvarez, M., Tuñón, M. J., et al. (2017). Inhibition of the SphK1/S1P Signaling Pathway by Melatonin in Mice with Liver Fibrosis and Human Hepatic Stellate Cells. Biofactors 43 (2), 272-282. doi:10.1002/biof.1342

Gu, Y. Y., Liu, X. S., Huang, X. R., Yu, X. Q., and Lan, H. Y. (2020). TGF-beta in Renal Fibrosis: Triumphs and Challenges. Future Med. Chem. 12 (9), 853-866. doi:10.4155/fmc-2020-0005

Guo, X., Cen, Y., Wang, J., and Jiang, H. (2018). CXCL10-induced IL-9 Promotes Liver Fibrosis via Raf/MEK/ERK Signaling Pathway. Biomed. Pharmacother. 105, 282-289. doi:10.1016/j.biopha.2018.05.128

Guo, D., Cheng, L., Shen, Y., Li, W., Li, Q., Zhong, Y., et al. (2020). 6-Bromoindirubin3'-oxime (6BIO) Prevents Myocardium from Aging by Inducing Autophagy. Aging (Albany NY) 12 (24), 26047-26062. doi:10.18632/aging.202253

Guzmán-Navarro, G., León, M. B., Martín-Estal, I., Durán, R. C., VillarrealAlvarado, L., Vaquera-Vázquez, A., et al. (2021). Prenatal Indole-3-Carbinol Administration Activates Aryl Hydrocarbon Receptor-Responsive Genes and Attenuates Lung Injury in a Bronchopulmonary Dysplasia Model. Exp. Biol. Med. (Maywood) 246 (6), 695-706. doi:10.1177/1535370220963789

Han, X., Zhang, Y. L., Fu, T. T., Li, P. B., Cong, T., and Li, H. H. (2020). Blockage of UCHL1 Activity Attenuates Cardiac Remodeling in Spontaneously Hypertensive Rats. Hypertens. Res. 43 (10), 1089-1098. doi:10.1038/s41440-020-0486-1

Hayashi, K., Shimokawa, T., Yamagata, M., and Yoneda, K. (2021). Inhibition of a2Adrenoceptor Is Renoprotective in 5/6 Nephrectomy-Induced Chronic Kidney Injury Rats. J. Pharmacol. Sci. 145 (1), 79-87. doi:10.1016/j.jphs.2020.11.001

Henderson, N. C., Rieder, F., and Wynn, T. A. (2020). Fibrosis: from Mechanisms to Medicines. Nature 587 (7835), 555-566. doi:10.1038/s41586-020-2938-9

Henriksbo, B. D., Lau, T. C., Cavallari, J. F., Denou, E., Chi, W., Lally, J. S., et al. (2014). Fluvastatin Causes NLRP3 Inflammasome-Mediated Adipose Insulin Resistance. Diabetes 63 (11), 3742-3747. doi:10.2337/db13-1398

Higashi, T., Friedman, S. L., and Hoshida, Y. (2017). Hepatic Stellate Cells as Key Target in Liver Fibrosis. Adv. Drug Deliv. Rev. 121, 27-42. doi:10.1016/j.addr.2017.05.007

Homo-Delarche, F., Calderari, S., Irminger, J. C., Gangnerau, M. N., Coulaud, J., Rickenbach, K., et al. (2006). Islet Inflammation and Fibrosis in a Spontaneous Model of Type 2 Diabetes, the GK Rat. Diabetes 55 (6), 1625-1633. doi:10.2337/ db05-1526

Horowitz, J. C., and Thannickal, V. J. (2019). Mechanisms for the Resolution of Organ Fibrosis. Physiology (Bethesda) 34 (1), 43-55. doi:10.1152/physiol.00033.2018

Insel, P. A., Murray, F., Yokoyama, U., Romano, S., Yun, H., Brown, L., et al. (2012). cAMP and Epac in the Regulation of Tissue Fibrosis. Br. J. Pharmacol. 166 (2), 447-456. doi:10.1111/j.1476-5381.2012.01847.x

Ishii, N., Araki, K., Yokobori, T., Hagiwara, K., Gantumur, D., Yamanaka, T., et al. (2019). Conophylline Suppresses Pancreatic Cancer Desmoplasia and CancerPromoting Cytokines Produced by Cancer-Associated Fibroblasts. Cancer Sci. 110 (1), 334-344. doi:10.1111/cas.13847

Ishikawa, H., Colby, D. A., Seto, S., Va, P., Tam, A., Kakei, H., et al. (2009). Total Synthesis of Vinblastine, Vincristine, Related Natural Products, and Key Structural Analogues. J. Am. Chem. Soc. 131 (13), 4904-4916. doi:10.1021/ja809842b

Jiang, J., Liang, S., Zhang, J., Du, Z., Xu, Q., Duan, J., et al. (2021). Melatonin Ameliorates PM2.5 -Induced Cardiac Perivascular Fibrosis through Regulating Mitochondrial Redox Homeostasis. J. Pineal Res. 70 (1), e12686. doi:10.1111/jpi.12686

Kang, J. W., Hong, J. M., and Lee, S. M. (2016). Melatonin Enhances Mitophagy and Mitochondrial Biogenesis in Rats with Carbon Tetrachloride-Induced Liver Fibrosis. J. Pineal Res. 60 (4), 383-393. doi:10.1111/jpi.12319

Karampitsakos, T., Woolard, T., Bouros, D., and Tzouvelekis, A. (2017). Toll-like Receptors in the Pathogenesis of Pulmonary Fibrosis. Eur. J. Pharmacol. 808, 35-43. doi:10.1016/j.ejphar.2016.06.045

Karimi-Shah, B. A., and Chowdhury, B. A. (2015). Forced Vital Capacity in Idiopathic Pulmonary Fibrosis--FDA Review of Pirfenidone and Nintedanib. N. Engl. J. Med. 372 (13), 1189-1191. doi:10.1056/NEJMp1500526
Katsuda, Y., Ohta, T., Miyajima, K., Kemmochi, Y., Sasase, T., Tong, B., et al. (2014). Diabetic Complications in Obese Type 2 Diabetic Rat Models. Exp. Anim. 63 (2), 121-132. doi:10.1538/expanim.63.121

Keglevich, P., Hazai, L., Kalaus, G., and Szántay, C. (2012). Modifications on the Basic Skeletons of Vinblastine and Vincristine. Molecules 17 (5), 5893-5914. doi: $10.3390 /$ molecules 17055893

Kendall, R. T., and Feghali-Bostwick, C. A. (2014). Fibroblasts in Fibrosis: Novel Roles and Mediators. Front. Pharmacol. 5, 123. doi:10.3389/fphar. 2014.00123

Kim, S. O., and Choi, Y. H. (2020). Indole-6-Carboxaldehyde Isolated from Sargassum Thunbergii (Mertens) Kuntze Prevents Oxidative Stress-Induced Cellular Damage in V79-4 Chinese Hamster Lung Fibroblasts through the Activation of the Nrf2/HO-1 Signaling Pathway. Cell Physiol Biochem 54 (5), 959-974. doi:10.33594/000000281

Kim, E. S., and Keating, G. M. (2015). Pirfenidone: A Review of its Use in Idiopathic Pulmonary Fibrosis. Drugs 75 (2), 219-230. doi:10.1007/s40265-015-0350-9

Kim, J. Y., Leem, J., and Jeon, E. J. (2019). Protective Effects of Melatonin against Aristolochic Acid-Induced Nephropathy in Mice. Biomolecules 10 (1), 11. doi:10.3390/biom10010011

Kong, P., Christia, P., and Frangogiannis, N. G. (2014). The Pathogenesis of Cardiac Fibrosis. Cell Mol Life Sci 71 (4), 549-574. doi:10.1007/s00018-013-1349-6

Kubo, N., Saito, R., Hamano, K., Nagasawa, M., Aoki, F., Takei, I., et al. (2014). Conophylline Suppresses Hepatic Stellate Cells and Attenuates ThioacetamideInduced Liver Fibrosis in Rats. Liver Int. 34 (7), 1057-1067. doi:10.1111/liv. 12328

Kurose, H., and Mangmool, S. (2016). Myofibroblasts and Inflammatory Cells as Players of Cardiac Fibrosis. Arch. Pharm. Res. 39 (8), 1100-1113. doi:10.1007/ s12272-016-0809-6

Leask, A. (2015). Getting to the Heart of the Matter: New Insights into Cardiac Fibrosis. Circ. Res. 116 (7), 1269-1276. doi:10.1161/circresaha.116.305381

Lebda, M. A., Sadek, K. M., Abouzed, T. K., Tohamy, H. G., and El-Sayed, Y. S. (2018). Melatonin Mitigates Thioacetamide-Induced Hepatic Fibrosis via Antioxidant Activity and Modulation of Proinflammatory Cytokines and Fibrogenic Genes. Life Sci. 192, 136-143. doi:10.1016/j.lfs.2017. 11.036

Lee, E., Ryu, G. R., Ko, S. H., Ahn, Y. B., and Song, K. H. (2017). A Role of Pancreatic Stellate Cells in Islet Fibrosis and $\beta$-Cell Dysfunction in Type 2 Diabetes Mellitus. Biochem. Biophys. Res. Commun. 485 (2), 328-334. doi:10. 1016/j.bbrc.2017.02.082

Lei, H., Guo, M., Li, X., Jia, F., Li, C., Yang, Y., et al. (2020). Discovery of Novel Indole-Based Allosteric Highly Potent ATX Inhibitors with Great In Vivo Efficacy in a Mouse Lung Fibrosis Model. J. Med. Chem. 63 (13), 7326-7346. doi:10.1021/acs.jmedchem.0c00506

Li, L. C., and Kan, L. D. (2017). Traditional Chinese Medicine for Pulmonary Fibrosis Therapy: Progress and Future Prospects. J. Ethnopharmacol 198, 45-63. doi:10.1016/j.jep.2016.12.042

Li, W. Q., Li, X. H., Du, J., Zhang, W., Li, D., Xiong, X. M., et al. (2016). Rutaecarpine Attenuates Hypoxia-Induced Right Ventricular Remodeling in Rats. Naunyn Schmiedebergs Arch. Pharmacol. 389 (7), 757-767. doi:10.1007/ s00210-016-1240-8

Li, B., Cong, M., Zhu, Y., Xiong, Y., Jin, W., Wan, Y., et al. (2017). Indole-3Carbinol Induces Apoptosis of Hepatic Stellate Cells through K63 DeUbiquitination of RIP1 in Rats. Cel Physiol Biochem 41 (4), 1481-1490. doi:10.1159/000470650

Li, J., Li, N., Yan, S., Lu, Y., Miao, X., Gu, Z., et al. (2019a). Melatonin Attenuates Renal Fibrosis in Diabetic Mice by Activating the AMPK/PGC1a Signaling Pathway and Rescuing Mitochondrial Function. Mol. Med. Rep. 19 (2), 1318-1330. doi:10.3892/mmr.2018.9708

Li, L., Ma, L., Wang, D., Jia, H., Yu, M., Gu, Y., et al. (2019b). Design and Synthesis of Matrine Derivatives as Novel Anti-Pulmonary Fibrotic Agents via Repression of the TGF $\beta /$ Smad Pathway. Molecules 24 (6), 1108. doi:10.3390/ molecules 24061108

Li, N., Wang, Z., Gao, F., Lei, Y., and Li, Z. (2020a). Melatonin Ameliorates Renal Fibroblast-Myofibroblast Transdifferentiation and Renal Fibrosis through miR21-5p Regulation. J. Cel Mol Med 24 (10), 5615-5628. doi:10.1111/jcmm.15221

Li, X., Ge, J., Zheng, Q., Zhang, J., Sun, R., and Liu, R. (2020b). Evodiamine and Rutaecarpine from Tetradium Ruticarpum in the Treatment of Liver Diseases. Phytomedicine 68, 153180. doi:10.1016/j.phymed.2020.153180 
Lin, P., Wang, M., Wei, Y., Kim, T., and Wei, X. (2020). Coronavirus in Human Diseases: Mechanisms and Advances in Clinical Treatment. MedComm (Beijing) 1 (3), 270-301. doi:10.1002/mco2.26

Liu, F., Wang, L., Qi, H., Wang, J., Wang, Y., Jiang, W., et al. (2017). Nintedanib, a Triple Tyrosine Kinase Inhibitor, Attenuates Renal Fibrosis in Chronic Kidney Disease. Clin. Sci. (Lond) 131 (16), 2125-2143. doi:10.1042/CS20170134

Liu, Q., Gao, Y., and Ci, X. (2019a). Role of Nrf2 and its Activators in Respiratory Diseases. Oxid Med. Cel Longev 2019, 7090534. doi:10.1155/2019/7090534

Liu, Z., Chen, L., Yu, P., Zhang, Y., Fang, B., Wu, C., et al. (2019b). Discovery of 3(Indol-5-Yl)-Indazole Derivatives as Novel Myeloid Differentiation Protein 2/ Toll-like Receptor 4 Antagonists for Treatment of Acute Lung Injury. J. Med. Chem. 62 (11), 5453-5469. doi:10.1021/acs.jmedchem.9b00316

Liu, J.-J., Shentu, L.-M., Ma, N., Wang, L.-Y., Zhang, G.-M., Sun, Y., et al. (2020). Inhibition of $\mathrm{NF}-\mathrm{Kb}$ and $\mathrm{Wnt} / \beta$-catenin/GSK3 $\beta$ Signaling Pathways Ameliorates Cardiomyocyte Hypertrophy and Fibrosis in Streptozotocin (STZ)-Induced Type 1 Diabetic Rats. Curr. Med. Sci. 40 (1), 35-47. doi:10. 1007/s11596-020-2144-x

Luo, M., Chen, L., Zheng, J., Wang, Q., Huang, Y., Liao, F., et al. (2021). Mitigation of Radiation-Induced Pulmonary Fibrosis by Small-Molecule Dye IR-780. Free Radic. Biol. Med. 164, 417-428. doi:10.1016/j.freeradbiomed.2020.12.435

Martins, D., and Nunez, C. V. (2015). Secondary Metabolites from Rubiaceae Species. Molecules 20 (7), 13422-13495. doi:10.3390/molecules200713422

Meng, X. M., Nikolic-Paterson, D. J., and Lan, H. Y. (2014). Inflammatory Processes in Renal Fibrosis. Nat. Rev. Nephrol. 10 (9), 493-503. doi:10.1038/ nrneph.2014.114

Meng, X. M., Tang, P. M., Li, J., and Lan, H. Y. (2015). TGF- $\beta /$ Smad Signaling in Renal Fibrosis. Front. Physiol. 6, 82. doi:10.3389/fphys.2015.00082

Mirzaei, S., Zarrabi, A., Hashemi, F., Zabolian, A., Saleki, H., Ranjbar, A., et al. (2021). Regulation of Nuclear Factor-KappaB (NF-Kb) Signaling Pathway by Non-coding RNAs in Cancer: Inhibiting or Promoting Carcinogenesis? Cancer Lett. 509, 63-80. doi:10.1016/j.canlet.2021.03.025

Montero, P., Milara, J., Roger, I., and Cortijo, J. (2021). Role of JAK/STAT in Interstitial Lung Diseases; Molecular and Cellular Mechanisms. Int. J. Mol. Sci. 22 (12), 6211. doi:10.3390/ijms22126211

Mori, M., Nakanishi, M., Kajishima, D., and Sato, Y. (2003). A Novel and General Synthetic Pathway to Strychnos Indole Alkaloids: Total Syntheses of (-)-tubifoline, (-)-dehydrotubifoline, and (-)-strychnine Using PalladiumCatalyzed Asymmetric Allylic Substitution. J. Am. Chem. Soc. 125 (32), 9801-9807. doi:10.1021/ja029382u

Mortezaee, K., Khanlarkhani, N., Sabbaghziarani, F., Nekoonam, S., Majidpoor, J., Hosseini, A., et al. (2017). Preconditioning with Melatonin Improves Therapeutic Outcomes of Bone Marrow-Derived Mesenchymal Stem Cells in Targeting Liver Fibrosis Induced by CCl4. Cell Tissue Res 369 (2), 303-312. doi:10.1007/s00441-017-2604-1

Novo, E., Cannito, S., Paternostro, C., Bocca, C., Miglietta, A., and Parola, M. (2014). Cellular and Molecular Mechanisms in Liver Fibrogenesis. Arch. Biochem. Biophys. 548, 20-37. doi:10.1016/j.abb.2014.02.015

Okumura, K., Kato, H., Honjo, O., Breitling, S., Kuebler, W. M., Sun, M., et al. (2015). Carvedilol Improves Biventricular Fibrosis and Function in Experimental Pulmonary Hypertension. J. Mol. Med. (Berl) 93 (6), 663-674. doi:10.1007/s00109-015-1251-9

Oruqaj, G., Karnati, S., Vijayan, V., Kotarkonda, L. K., Boateng, E., Zhang, W., et al. (2015). Compromised Peroxisomes in Idiopathic Pulmonary Fibrosis, a Vicious Cycle Inducing a Higher Fibrotic Response via TGF- $\beta$ Signaling. Proc. Natl. Acad. Sci. U S A. 112 (16), E2048-E2057. doi:10.1073/pnas.1415111112

Panda, S., Kar, A., and Ramamurthy, V. (2014). Cardioprotective Effect of Vincristine on Isoproterenol-Induced Myocardial Necrosis in Rats. Eur. J. Pharmacol. 723, 451-458. doi:10.1016/j.ejphar.2013.10.049

Pang, Q., Jin, H., Wang, Y., Dai, M., Liu, S., Tan, Y., et al. (2021). Depletion of Serotonin Relieves Concanavalin A-Induced Liver Fibrosis in Mice by Inhibiting Inflammation, Oxidative Stress, and TGF- $\beta 1 /$ Smads Signaling Pathway. Toxicol. Lett. 340, 123-132. doi:10.1016/j.toxlet.2021.01.010

Parola, M., and Pinzani, M. (2019). Liver Fibrosis: Pathophysiology, Pathogenetic Targets and Clinical Issues. Mol. Aspects Med. 65, 37-55. doi:10.1016/j.mam. 2018.09.002

Pinto, A. R., Ilinykh, A., Ivey, M. J., Kuwabara, J. T., D’Antoni, M. L., Debuque, R., et al. (2016). Revisiting Cardiac Cellular Composition. Circ. Res. 118 (3), 400-409. doi:10.1161/circresaha.115.307778
Pompili, S., Sferra, R., Gaudio, E., Viscido, A., Frieri, G., Vetuschi, A., et al. (2019). Can Nrf2 Modulate the Development of Intestinal Fibrosis and Cancer in Inflammatory Bowel Disease? Int. J. Mol. Sci. 20 (16), 4061. doi:10.3390/ijms20164061

Pourheydar, B., Samadi, M., Habibi, P., Nikibakhsh, A. A., and Naderi, R. (2021). Renoprotective Effects of Tropisetron through Regulation of the TGF- $\beta 1$, P53 and Matrix Metalloproteinases in Streptozotocin-Induced Diabetic Rats. Chem. Biol. Interact 335, 109332. doi:10.1016/j.cbi.2020.109332

Qian, J., Yin, S., Ye, L., Wang, Z., Shu, S., Mou, Z., et al. (2021). An Indole-2Carboxamide Derivative, LG4, Alleviates Diabetic Kidney Disease through Inhibiting MAPK-Mediated Inflammatory Responses. J. Inflamm. Res. 14, 1633-1645. doi:10.2147/JIR.S308353

Qiu, M., Yang, Z., Bian, M., Liu, C., Zhao, Y., and Liu, Q. (2020). Protective Effects of Isorhynchophylline against Silicon-Dioxide-Induced Lung Injury in Mice. Artif. Cell Nanomed Biotechnol 48 (1), 1125-1134. doi:10.1080/21691401.2020.1814315

Rajasekaran, S., Rajaguru, P., and Sudhakar Gandhi, P. S. (2015). MicroRNAs as Potential Targets for Progressive Pulmonary Fibrosis. Front. Pharmacol. 6, 254. doi:10.3389/fphar.2015.00254

Rao, Y., Lu, Y. T., Li, C., Song, Q. Q., Xu, Y. H., Xu, Z., et al. (2019). Bouchardatine Analogue Alleviates Non-alcoholic Hepatic Fatty Liver Disease/Non-Alcoholic Steatohepatitis in High-Fat Fed Mice by Inhibiting ATP Synthase Activity. Br. J. Pharmacol. 176 (16), 2877-2893. doi:10.1111/bph.14713

Richter, K., Konzack, A., Pihlajaniemi, T., Heljasvaara, R., and Kietzmann, T. (2015). Redox-fibrosis: Impact of TGF $\beta 1$ on ROS Generators, Mediators and Functional Consequences. Redox Biol. 6, 344-352. doi:10.1016/j.redox.2015.08.015

Rienks, M., Papageorgiou, A. P., Frangogiannis, N. G., and Heymans, S. (2014). Myocardial Extracellular Matrix: an Ever-Changing and Diverse Entity. Circ. Res. 114 (5), 872-888. doi:10.1161/circresaha.114.302533

Rockey, D. C., Bell, P. D., and Hill, J. A. (2015). Fibrosis - A Common Pathway to Organ Injury and Failure. N. Engl. J. Med. 372 (12), 1138-1149. doi:10.1056/ NEJMra1300575

Said, R., and Tsimberidou, A. M. (2014). Pharmacokinetic Evaluation of Vincristine for the Treatment of Lymphoid Malignancies. Expert Opin. Drug Metab. Toxicol. 10 (3), 483-494. doi:10.1517/17425255.2014.885016

Saito, R., Yamada, S., Yamamoto, Y., Kodera, T., Hara, A., Tanaka, Y., et al. (2012). Conophylline Suppresses Pancreatic Stellate Cells and Improves Islet Fibrosis in Goto-Kakizaki Rats. Endocrinology 153 (2), 621-630. doi:10.1210/en.2011-1767

Sanchez-Valle, V., C. Chavez-Tapia, N., Uribe, M., and Mendez-Sanchez, N. (2012). Role of Oxidative Stress and Molecular Changes in Liver Fibrosis: A Review. Curr. Med. Chem. 19 (28), 4850-4860. doi:10.2174/092986712803341520

Santos, L. S., Theoduloz, C., Pilli, R. A., and Rodriguez, J. (2009). Antiproliferative Activity of Arborescidine Alkaloids and Derivatives. Eur. J. Med. Chem. 44 (9), 3810-3815. doi:10.1016/j.ejmech.2009.04.005

Sasaki, Y., Kato, D., and Boger, D. L. (2010). Asymmetric Total Synthesis of Vindorosine, Vindoline, and Key Vinblastine Analogues. J. Am. Chem. Soc. 132 (38), 13533-13544. doi:10.1021/ja106284s

Schunk, S. J., Floege, J., Fliser, D., and Speer, T. (2021). WNT- $\beta$-Catenin Signalling - a Versatile Player in Kidney Injury and Repair. Nat. Rev. Nephrol. 17 (3), 172-184. doi:10.1038/s41581-020-00343-w

Schwensen, H. F., Borreschmidt, L. K., Storgaard, M., Redsted, S., Christensen, S., and Madsen, L. B. (2020). Fatal Pulmonary Fibrosis: a post-COVID-19 Autopsy Case. J. Clin. Pathol. 74, 400-402. doi:10.1136/jclinpath-2020-206879

Sehgal, R., Ilha, M., Vaittinen, M., Kaminska, D., Männistö, V., Kärjä, V., et al. (2021). Indole-3-Propionic Acid, a Gut-Derived Tryptophan Metabolite, Associates with Hepatic Fibrosis. Nutrients 13 (10), 3509. doi:10.3390/nu13103509

Shima, H., Sasaki, K., Suzuki, T., Mukawa, C., Obara, T., Oba, Y., et al. (2017). A Novel Indole Compound MA-35 Attenuates Renal Fibrosis by Inhibiting Both TNF- $\alpha$ and TGF- $\beta 1$ Pathways. Sci. Rep. 7, 1884. doi:10.1038/s41598-017-01702-7

Singh, T. P., and Singh, O. M. (2018). Recent Progress in Biological Activities of Indole and Indole Alkaloids. Mini Rev. Med. Chem. 18 (1), 9-25. doi:10.2174/ 1389557517666170807123201

Song, Y. J., Zhong, C. B., and Wu, W. (2020). Cardioprotective Effects of Melatonin: Focusing on its Roles against Diabetic Cardiomyopathy. Biomed. Pharmacother. 128, 110260. doi:10.1016/j.biopha.2020.110260

Soria, J. C., Ohe, Y., Vansteenkiste, J., Reungwetwattana, T., Chewaskulyong, B., Lee, K. H., et al. (2018). Osimertinib in Untreated EGFR-Mutated Advanced Non-SmallCell Lung Cancer. N. Engl. J. Med. 378 (2), 113-125. doi:10.1056/NEJMoa1713137 Sravanthi, T. V., and Manju, S. L. (2016). Indoles - A Promising Scaffold for Drug Development. Eur. J. Pharm. Sci. 91, 1-10. doi:10.1016/j.ejps.2016.05.025 
Sun, Y. B., Qu, X., Caruana, G., and Li, J. (2016). The Origin of Renal Fibroblasts/ Myofibroblasts and the Signals that Trigger Fibrosis. Differentiation 92 (3), 102-107. doi:10.1016/j.diff.2016.05.008

Tian, S., Xiong, Y., Liu, H., Niu, L., Guo, J., Liao, M., et al. (2020). Pathological Study of the 2019 Novel Coronavirus Disease (COVID-19) through Postmortem Core Biopsies. Mod. Pathol. 33 (6), 1007-1014. doi:10.1038/s41379-020-0536-x

Todd, N. W., Luzina, I. G., and Atamas, S. P. (2012). Molecular and Cellular Mechanisms of Pulmonary Fibrosis. Fibrogenesis Tissue Repair 5 (1), 11. doi:10. $1186 / 1755-1536-5-11$

Umemura, Y., Mitsuyama, Y., Minami, K., Nishida, T., Watanabe, A., Okada, N., et al. (2021). Efficacy and Safety of Nintedanib for Pulmonary Fibrosis in Severe Pneumonia Induced by COVID-19: An Interventional Study. Int. J. Infect. Dis. 108, 454-460. doi:10.1016/j.ijid.2021.05.055

van Nieuwenhoven, F. A., and Turner, N. A. (2013). The Role of Cardiac Fibroblasts in the Transition from Inflammation to Fibrosis Following Myocardial Infarction. Vascul Pharmacol. 58 (3), 182-188. doi:10.1016/j.vph.2012.07.003

Wang, M., Chen, D. Q., Chen, L., Cao, G., Zhao, H., Liu, D., et al. (2018). Novel Inhibitors of the Cellular Renin-Angiotensin System Components, Poricoic Acids, Target Smad3 Phosphorylation and Wnt/ $\beta$-Catenin Pathway against Renal Fibrosis. Br. J. Pharmacol. 175 (13), 2689-2708. doi:10.1111/bph.14333

Wang, Q., Yu, J., Hu, Y., Chen, X., Zhang, L., Pan, T., et al. (2020). Indirubin Alleviates Bleomycin-Induced Pulmonary Fibrosis in Mice by Suppressing Fibroblast to Myofibroblast Differentiation. Biomed. Pharmacother. 131, 110715. doi:10.1016/j.biopha.2020.110715

Wei, Y., Kang, X. L., and Wang, X. (2014). The Peripheral Cannabinoid Receptor 1 Antagonist VD60 Efficiently Inhibits Carbon Tetrachloride-Intoxicated Hepatic Fibrosis Progression. Exp. Biol. Med. (Maywood) 239 (2), 183-192. doi:10.1177/1535370213514922

Weiskirchen, R., Weiskirchen, S., and Tacke, F. (2019). Organ and Tissue Fibrosis: Molecular Signals, Cellular Mechanisms and Translational Implications. Mol. Aspects Med. 65, 2-15. doi:10.1016/j.mam.2018.06.003

Wollin, L., Wex, E., Pautsch, A., Schnapp, G., Hostettler, K. E., Stowasser, S., et al. (2015). Mode of Action of Nintedanib in the Treatment of Idiopathic Pulmonary Fibrosis. Eur. Respir. J. 45 (5), 1434-1445. doi:10.1183/09031936.00174914

Wu, M. P., Zhang, Y. S., Xu, X., Zhou, Q., Li, J. D., and Yan, C. (2017a). Vinpocetine Attenuates Pathological Cardiac Remodeling by Inhibiting Cardiac Hypertrophy and Fibrosis. Cardiovasc. Drugs Ther. 31 (2), 157-166. doi:10. 1007/s10557-017-6719-0

Wu, Q. Q., Xiao, Y., Jiang, X. H., Yuan, Y., Yang, Z., Chang, W., et al. (2017b). Evodiamine Attenuates TGF- $\beta 1$-Induced Fibroblast Activation and Endothelial to Mesenchymal Transition. Mol. Cel Biochem 430 (1-2), 81-90. doi:10.1007/ s11010-017-2956-6

Wu, Y., Li, Z., Wang, S., Xiu, A., and Zhang, C. (2019). Carvedilol Inhibits Angiotensin II-Induced Proliferation and Contraction in Hepatic Stellate Cells through the RhoA/Rho-Kinase Pathway. Biomed. Res. Int. 2019, 7932046. doi:10.1155/2019/7932046

Wynn, T. A., and Ramalingam, T. R. (2012). Mechanisms of Fibrosis: Therapeutic Translation for Fibrotic Disease. Nat. Med. 18 (7), 1028-1040. doi:10.1038/nm.2807

Xia, Z. E., Xi, J. L., and Shi, L. (2018). 3,3'-Diindolylmethane Ameliorates Renal Fibrosis through the Inhibition of Renal Fibroblast Activation In Vivo and In Vitro. Ren. Fail. 40 (1), 447-454. doi:10.1080/0886022X.2018.1490322

Xue, R., Jia, K., Wang, J., Yang, L., Wang, Y., Gao, L., et al. (2018). A Rising Star in Pancreatic Diseases: Pancreatic Stellate Cells. Front. Physiol. 9, 754. doi:10. 3389/fphys.2018.00754

Yang, D., Li, L., Qian, S., and Liu, L. (2018). Evodiamine Ameliorates Liver Fibrosis in Rats via TGF- $\beta 1 /$ Smad Signaling Pathway. J. Nat. Med. 72 (1), 145-154. doi:10.1007/s11418-017-1122-5

Yao, Z., Hu, W., Yin, S., Huang, Z., Zhu, Q., Chen, J., et al. (2013). 3,3'Diindolymethane Ameliorates Adriamycin-Induced Cardiac Fibrosis via Activation of a BRCA1-dependent Anti-oxidant Pathway. Pharmacol. Res. 70 (1), 139-146. doi:10.1016/j.phrs.2013.01.006

Ye, C., Zhang, N., Zhao, Q., Xie, X., Li, X., Zhu, H. P., et al. (2021). Evodiamine Alleviates Lipopolysaccharide-Induced Pulmonary Inflammation and Fibrosis by Activating Apelin Pathway. Phytother Res. 35 (6), 3406-3417. doi:10.1002/ptr.7062

Yoon, Y. M., Go, G., Yun, C. W., Lim, J. H., Lee, J. H., and Lee, S. H. (2020). Melatonin Suppresses Renal Cortical Fibrosis by Inhibiting Cytoskeleton Reorganization and Mitochondrial Dysfunction through Regulation of miR4516. Int. J. Mol. Sci. 21 (15), 5323. doi:10.3390/ijms21155323
Yu, H., Lin, L., Zhang, Z., Zhang, H., and Hu, H. (2020). Targeting NF-Kb Pathway for the Therapy of Diseases: Mechanism and Clinical Study. Signal. Transduct Target. Ther. 5 (1), 209. doi:10.1038/s41392-020-00312-6

Zahran, M. A. H., El-Aarag, B., Mehany, A. B. M., Belal, A., and Younes, A. S. (2018). Design, Synthesis, Biological Evaluations, Molecular Docking, and In Vivo Studies of Novel Phthalimide Analogs. Arch. Pharm. (Weinheim) 351 (5), e1700363. doi:10.1002/ardp.201700363

Zhang, Y. D., Ding, X. J., Dai, H. Y., Peng, W. S., Guo, N. F., Zhang, Y., et al. (2018). SB-216763, a GSK-3 $\beta$ Inhibitor, Protects against Aldosterone-Induced Cardiac, and Renal Injury by Activating Autophagy. J. Cel Biochem 119 (7), 5934-5943. doi:10.1002/jcb.26788

Zhang, T., Wang, X. F., Wang, Z. C., Lou, D., Fang, Q. Q., Hu, Y. Y., et al. (2020a). Current Potential Therapeutic Strategies Targeting the TGF- $\beta /$ Smad Signaling Pathway to Attenuate Keloid and Hypertrophic Scar Formation. Biomed. Pharmacother. 129, 110287. doi:10.1016/j.biopha.2020.110287

Zhang, Y., Cui, Y., Dai, S., Deng, W., Wang, H., Qin, W., et al. (2020b). Isorhynchophylline Enhances Nrf2 and Inhibits MAPK Pathway in Cardiac Hypertrophy. Naunyn Schmiedebergs Arch. Pharmacol. 393 (2), 203-212. doi:10.1007/s00210-019-01716-0

Zhang, Y., Zou, J., Tolbert, E., Zhao, T. C., Bayliss, G., and Zhuang, S (2020c). Identification of Histone Deacetylase 8 as a Novel Therapeutic Target for Renal Fibrosis. FASEB J. 34 (6), 7295-7310. doi:10.1096/fj.201903254R

Zhangdi, H. J., Su, S. B., Wang, F., Liang, Z. Y., Yan, Y. D., Qin, S. Y., et al. (2019). Crosstalk Network Among Multiple Inflammatory Mediators in Liver Fibrosis. World J. Gastroenterol. 25 (33), 4835-4849. doi:10.3748/wjg.v25.i33.4835

Zhao, Y. L., Yang, Z. F., Wu, B. F., Shang, J. H., Liu, Y. P., Wang, X. H., et al. (2020). Indole Alkaloids from Leaves of Alstonia S (L.) R. Br. Protect against Emphysema in Mice. J. Ethnopharmacol 259, 112949. doi:10.1016/j.jep.2020.112949

Zhao, Y. L., Pu, S. B., Qi, Y., Wu, B. F., Shang, J. H., Liu, Y. P., et al. (2021). Pharmacological Effects of Indole Alkaloids from Alstonia S (L.) R. Br. On Pulmonary Fibrosis In Vivo. J. Ethnopharmacol 267, 113506. doi:10.1016/j.jep.2020.113506

Zheng, W., Shang, X., Zhang, C., Gao, X., Robinson, B., and Liu, J. (2017). The Effects of Carvedilol on Cardiac Function and the AKT/XIAP Signaling Pathway in Diabetic Cardiomyopathy Rats. Cardiology 136 (3), 204-211. doi:10.1159/000450825

Zhou, J. Y., and Zhou, S. W. (2012). Isorhynchophylline: A Plant Alkaloid with Therapeutic Potential for Cardiovascular and central Nervous System Diseases. Fitoterapia 83 (4), 617-626. doi:10.1016/j.fitote.2012.02.010

Zhou, H., Yue, Y., Wang, J., Ma, Q., and Chen, Y. (2018). Melatonin Therapy for Diabetic Cardiomyopathy: A Mechanism Involving Syk-Mitochondrial Complex I-SERCA Pathway. Cell Signal 47, 88-100. doi:10.1016/j.cellsig.2018.03.012

Zhou, S., Wang, Y., Zhu, T., and Xia, L. (2020). CT Features of Coronavirus Disease 2019 (COVID-19) Pneumonia in 62 Patients in Wuhan, China. AJR Am. J. Roentgenol 214 (6), 1287-1294. doi:10.2214/ajr.20.22975

Zhu, J. N., Chen, R., Fu, Y. H., Lin, Q. X., Huang, S., Guo, L. L., et al. (2013). Smad3 Inactivation and MiR-29b Upregulation Mediate the Effect of Carvedilol on Attenuating the Acute Myocardium Infarction-Induced Myocardial Fibrosis in Rat. Plos One 8 (9), e75557. doi:10.1371/journal.pone.0075557

Zhu, J., Wang, M., Wen, W., and Yu, R. (2015). Biosynthesis and Regulation of Terpenoid Indole Alkaloids in Catharanthus Roseus. Pharmacogn Rev. 9 (17), 24-28. doi:10.4103/0973-7847.156323

Conflict of Interest: The authors declare that the research was conducted in the absence of any commercial or financial relationships that could be construed as a potential conflict of interest.

Publisher's Note: All claims expressed in this article are solely those of the authors and do not necessarily represent those of their affiliated organizations, or those of the publisher, the editors and the reviewers. Any product that may be evaluated in this article, or claim that may be made by its manufacturer, is not guaranteed or endorsed by the publisher.

Copyright (c) 2022 Qin, Zhao, Han, Zhu, Peng, Zhan and Huang. This is an openaccess article distributed under the terms of the Creative Commons Attribution License (CC BY). The use, distribution or reproduction in other forums is permitted, provided the original author(s) and the copyright owner(s) are credited and that the original publication in this journal is cited, in accordance with accepted academic practice. No use, distribution or reproduction is permitted which does not comply with these terms. 